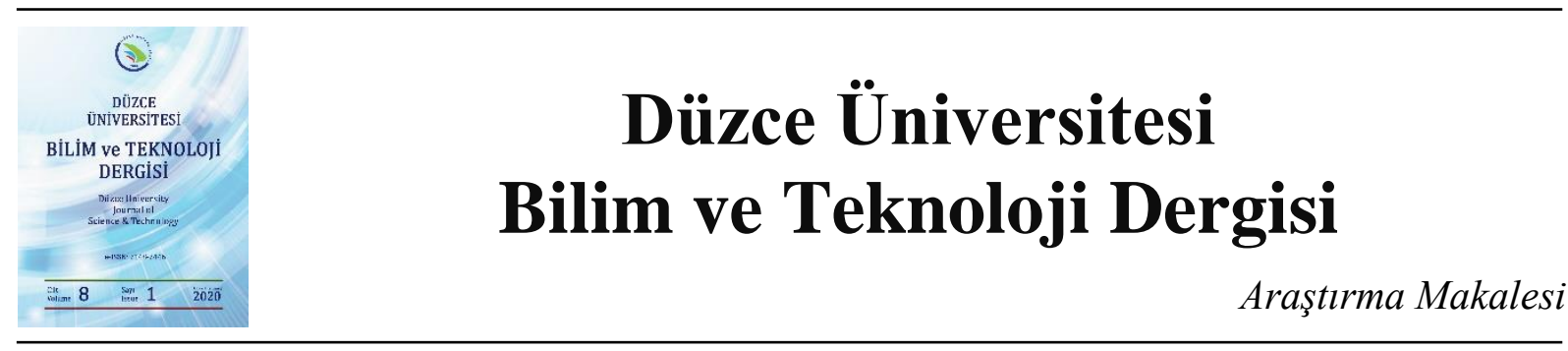

\section{Rulet Elektromanyetik Alan Optimizasyon (R-EFO) Algoritması}

\author{
(iD) Hamdi Tolga KAHRAMAN ${ }^{\mathrm{a}, *}$ \\ a," Yazllım Mühendisliği Bölümü, Of Teknoloji Fakültesi, Karadeniz Teknik Üniversitesi, Trabzon, TÜRKIYYE \\ * Sorumlu yazarin e-posta adresi: htolgakahraman@ktu.edu.tr
}

DOI : 10.29130/dubited.589259

\begin{abstract}
ÖZET
Meta-sezgisel optimizasyon algoritmalarının yerel arama performansları üzerinde etkili olan iki temel öğe seçim yöntemleri ve arama operatörleridir. Bu makale çalışmasında olasılıksal bir seçim yöntemi olan rulet tekerleğinin güncel bir meta-sezgisel arama tekniği olan elektromanyetik alan optimizasyon (electromagnetic field optimization, EFO) algoritmasının yerel arama performansı üzerindeki etkisi araştırılmaktadır. Elektromanyetik optimizasyon algoritmasında çözüm adayları topluluğu uygunluk değerlerine bağlı olarak pozitif, nötr ve negatif alanlara ayrılmaktadır. Bu üç alandan seçilen çözüm adayları ise arama sürecine rehberlik etmektedirler. Bu süreçte çözüm adayları açgözlü ve rastgele seçim yöntemleri ile belirlenmektedir. Bu makale çalışmasında ise negatif alandan çözüm adaylarının seçimi için rulet tekniği kullanılmaktadır. Deneysel çalı̧̧malarda literatürdeki en güncel sürekli değer problemleri olan CEC17 test seti kullanılmıştır. Deneysel çalı̧̧ma sonuçları istatistiksel olarak ikili karşılaştırmalarda kullanılan wilcoxon runk sum test ile analiz edilmiştir. Analiz sonuçlarına göre rulet seçim yöntemi EFO algoritmasının arama performansını kayda değer şekilde artırmaktadır.
\end{abstract}

Anahtar Kelimeler: Elektromanyetik alan optimizasyon algoritması, Rulet seçim yöntemi, CEC17 karşılaştırma problemleri, Meta-sezgisel optimizasyon

\section{Roulette Electromagnetic Field Optimization (R-EFO) Algorithm}

\begin{abstract}
Two key elements that influence the local search performance of meta-heuristic optimization algorithms are selection methods and search operators. In this article, the effect of roulette wheel, which is a probabilistic selection method, on local search performance of EFO (electromagnetic field optimization) algorithm, which is a current meta-heuristic search technique, is researched. In the EFO, the group of solution candidates are divided into positive, neutral and negative fields depending on their fitness values. The solution candidates selected from these three fields guide the search process. In this process, solution candidates are determined by greedy and random selection methods. In this study, the roulette technique is used for selection of solution candidates from negative field. In the experimental studies, the continuous valued and unconstrained problems CEC17 benchmark suite are used to test the performance of proposed Roulette- Electromagnetic Field Optimization (R-EFO). The results of the experimental study are statistically analyzed by Wilcoxon runk sum test used in comparison with standard EFO algorithm. According to the analysis results, proposed R-EFO algorithm with roulette selection method significantly improves the search performance of the EFO algorithm.
\end{abstract}

Keywords: Electromagnetic field optimization, Roulette selection, CEC17 benchmark suite, Meta-heuristic optimization 


\section{GiRis}

Meta-sezgisel arama (MSA) algoritmaları başta optimizasyon problemlerinin çözümlenmesi olmak üzere melez yapay zeka algoritmalarının geliştirilmesinde de yaygın olarak kullanılan güçlü ve etkili yöntemlerdir [1-9]. 1950'li yıllardan bu yana yüzlerce yeni MSA algoritması geliştirilmiş ve binlerce optimizasyon çalışmasına tatbik edilmiştir [10-14]. MSA algoritmalarının arama performanslarının iyileştirilmesi üzerine ise çok daha fazla çalışma yapılmıştır [12-13]. Bu çalışmaların birçoğu mevcut MSA tekniklerinin çeşitli yöntemlerle iyileştirilmesini ve varyasyonlarının geliştirilmesini konu almaktadır. MSA algoritmalarının arama performansları üzerinde etkili olan iki temel öğe seçim yöntemleri ve arama operatörleridir. Seçim yöntemleri ile arama sürecine rehberlik eden çözüm adayları belirlenmektedir. MSA algoritmalarında arama süreci yaşam döngüsü üç adımdan oluşmaktadır [1213]. Bunlar:

i) Seçim süreci: arama sürecinin yöneleceği çözüm aday(lar)ının belirlenmesi (seçimi)

ii) Komşuluk aramasının gerçekleştirilmesi: seçilen çözüm aday/adaylarının yakın çevresinde yapılan arama

iii) Çeşitliliğin sağlanması: seçilen/yeni üretilen çözüm aday/adaylarından faydalanılarak üretilen çözüm aday(lar)1

Yukarıda verilen arama döngüsü incelendiğinde sürecin ilk adımı olan seçim yönteminden elde edilen çıktıların (çözüm aday(lar)ının) diğer iki adımın da girdisi olduğu ve onların başarısını doğrudan etkilediği anlaşılmaktadır. Seçim yöntemi bu yönüyle arama sürecinin tamamını etkilemektedir (Cui vd. 2018 [15]). Seçim yöntemleri için bazı önemli değerlendirmeleri yapmak mümkündür. Seçim yöntemleri MSA sürecinin geneli ve nihai başarısı üzerinde doğrudan etkilidir. Tamamen rastgeleliğe dayalı seçim yöntemlerinin kullanıldığı MSA süreçlerinde yüksek karmaşıklık düzeyine sahip problemler için başarı elde etmek olanaksız hale gelebilmektedir. Açgözlü yöntemi benimseyen ve çözüm adaylarının sadece uygunluk değerlerine göre seçim işlemini gerçekleştiren MSA süreçlerinde yerel en iyi çözüm tuzaklarına takılma olasılığı artmaktadır. Bu durum hızlı yakınsama şeklinde de görülebilmektedir. Bu aşamada yerel çözüm tuzaklarına yakınsamayı önleyebilecek mekanizmalardan biri çeşitlilik operatörüdür. Özellikle çok boyutlu karmaşı arama uzaylarındaki yerel çözüm tuzakları en başarılı çeşitlilik operatörleri için dahi çözülmesi oldukça zor bir problemdir. Olasılıksal seçim yöntemleri çeşitliliği desteklemesi bakımından deterministtik ve rastgele seçim yöntemlerine göre daha etkili rehberlik edebilmektedirler.

EFO, elektromıknatısların farklı kutuplarının davranışlarından esinlenilerek 2016 yılında geliştirilmiş etkili bir MSA algoritmasıdır. EFO'da elektromıknatıslardan oluşan elektromanyetik parçacıklar çözüm adaylarını temsil etmektedirler. Bu parçacıklardan oluşan çözüm adayları topluluğu parçacıkların uygunluk değerlerine bağlı olarak üç alana (pozitif, negatif ve nötr) bölünmektedir. Arama sürecinde bu üç alandan seçilen elektromıknatıslar arasındaki itme ve çekme kuvvetleri parçacıkların konumlarını değiştirmekte ve genel çözüme yakınsamayı sağlamaktadır. Bu makale çalışmasında çözüm adaylarının uygunluk değerlerini seçilme olasılıklarına dönüştüren rulet tekerleği, güncel bir MSA tekniği olan EFO algoritmasına tatbik edilmektedir. Çalışmanın amacı rulet seçim yönteminin EFO algoritmasının komşuluk araması ve çeşitlilik performansı üzerindeki etkisini araştırmak ve genel olarak arama performans1 iyileştirmektir. Deneysel çalışmalarda 30 adet sürekli değer optimizasyon probleminden oluşan CEC17 test seti kullanılmıştır [16]. Deneysel çalışma sonuçları istatistiksel olarak wilcoxon runk sum test ile analiz edilmiş ve rulet seçim yönteminin EFO algoritmasının arama performansını iyileştirdiği görülmüştür. 


\section{YÖNTEM}

\section{A. SEÇİM YÖNTEMLERİ VE RULET TEKERLEĞİ}

MSA algoritmasında arama döngüsü üç adımdan oluşmaktadır. Bunlar arama operatörlerinin referans alacakları ve arama sürecine rehberlik edecek olan çözüm adayı seçim süreci, komşuluk araması ve çeşitlilik adımlarıdır. Bu üç adımın somut bir örneği olarak genetik algoritma [10] incelenecek olursa sürecin ilk adımı ebeveyn seçimidir. MSA algoritmaları topluluk tabanlı dolayısıyla paralel arama yapan teknikler olduklarından genetik algoritmada ebeveyn seçimi de haliyle çözüm adayları topluluğu (popülasyon) içerisinden yapılmaktadır. Bu amaçla başlıca üç seçim yöntemi kullanılmaktadır. Bunların ilki topluluk içerisinden tamamen rastgele seçim yapma şeklinde, ikincisi çözüm adaylarının sadece uygunluk değerlerine bakılarak seçim yapma şeklinde ve üçüncüsü de rulet tekerleği olarak adlandırılan ve çözüm adaylarının seçilme olasılığını uygunluk değerlerine bağlı olarak belirleyen tekniği kullanarak seçim yapma şeklindedir [18-20]. Bunların dışında ikili turnuva gibi farklı seçim teknikleri olsa da bunları üç sınıfta toplamak mümkündür. Bunlar deterministtik olmayan (tamamen rastgele gerçekleşen), deterministtik olan (tamamen uygunluk değerine bağlı olarak gerçekleşen) ve karma seçim yöntemleri (uygunluk değerini ve rastgeleliği dikkate alarak gerçekleşen) olarak özetlenebilir [18-22]. Genetik algoritma dışındaki MSA algoritmalarında seçim yöntemi olarak en çok deterministtik olan yöntem kullanılmaktadır [1-5, 23-30]. Bu yöntemin uygulanışı topluluk içerisinde uygunluk değeri en yüksek olan çözüm adayının seçilmesidir. İster deterministtik olsun isterse de karma yöntem olsun seçim sürecinde çözüm adaylarının uygunluk değerleri referans alınmaktadır [18-22]. Bu durum MSA sürecinin en iyi çözüm aday(lar)ına hızlıca yakınsamasına neden olabilmektedir. Hızlı yakınsama problemi ise arama sürecinin özellikle üçüncü adımını yerine getiren çeşitlilik operatörü/işlevi yardımıyla çözülmeye çalışılmaktadır. Ancak üçüncü adımda ifade edildiği gibi çeşitlilik operatörü birinci adımda seçilen birey (birinci senaryo) üzerinden ya da yeni/rastgele üretilen çözüm adayı (ikinci senaryo) üzerinden işletilen bir süreçtir. İlk senaryonun başarısının birinci adımda seçilen bireye bağlı olduğu çok açıktır. Çünkü birinci adımda seçilen bireyi kullanmaktadır. İkinci senaryo (esasında tamamen rastgele arama) ise başarı ihtimali çok düşük olduğundan (özellikle çok boyutlu ya da büyük arama uzaylarında) zaten çok az algoritma tarafından kullanılmaktadır. Hal böyle iken farklı MSA tekniklerinin çeşitlilik operatörlerinin işleyişleri ve yetenekleri birbirlerinden çok farklı da olsa hepsinin ortak yanı birinci adımda seçilen çözüm aday(lar)ı üzerinde işlem yapmalarıdır. Seçim sürecinde ise çözüm adaylarının uygunluk değerlerinin belirleyici olması algoritmaların hızlı yakınsamasına, yerel en iyi çözüm tuzaklarına takılmasına dolayısıyla genel (küresel) en iyi çözümü bulamamasına neden olabilmektedir. Olasılıksal seçim yöntemleri, açgözlü ve rastgele seçim yöntemlerine karşın arama sürecini yerel çözüm tuzaklarından daha güçlü bir şekilde kurtarma yeteneğine sahiptir. Devamlı olarak açgözlü yöntem ile seçilen rehber konumlar arama süreci yaşam döngüsünün ilerleyen adımlarında prematüre yakınsama problemine neden olmaktadır. Yani topluluk içerisindeki tüm adayların en iyi çözüm adayına benzemesine ve bir süre sonra arama uzayında birbirine çok yakın hatta aynı konumu temsil eden çözüm adayları haline dönüşmeleri söz konusudur. Buna karşın topluluk içerisinden rastgele seçim yapmak ise arama sürecinin ilerleyen adımlarında rastgele aramaya dönüşmekte ve global çözüm için gereken hassas komşuluk aramasına engel olmaktadır. Olasılıksal seçim yöntemleri ise her iki problemin çözümünde de etkili olmaktadır. Bir taraftan çözüm adaylarının uygunluk değerlerine bağlı olarak seçilme olasılıkları artarken komşuluk araması sürecinde güçlü adayların rehber seçilmesi sağlanmakta diğer taraftan rastgelelik sayesinde devamlı olarak en iyi çözümün rehber alınması engellenerek prematüre yakınsama problemine de önlem alınmaktadır. 


\section{Algoritma 1. Rulet seçim yöntemi sözde kodu}

n: MSA algoritmasında çözüm adayı sayısı, $\mathrm{m}=$ Optimizasyon probleminin boyutu

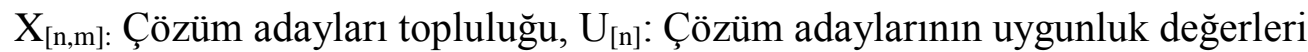

$\mathrm{R}_{[\mathrm{n}]}$ : Çözüm adaylarının rulet tekerleği yüzdeleri, $\mathrm{K}_{[\mathrm{n}]}$ : Çözüm adaylarının rulet tekerleği

konumlar1,

$\mathrm{t}=0, \mathrm{~K}_{[0]}=0$;

for $\mathrm{i}=1: \mathrm{n}$

$$
t=t+U_{[i]}
$$

end

for $i=1: n$

$\mathrm{R}_{[\mathrm{i}]}=\mathrm{U}_{[\mathrm{i}]} / \mathrm{t}$

$\mathrm{K}_{[\mathrm{i}]}=\mathrm{R}_{[\mathrm{i}]}+\mathrm{K}_{[\mathrm{i}-1]}$

end

konum=rand $(\mathbf{0 , 1}) * t / /$ rulet tekerleğini döndür ve tekerleğin durduğu konumu

belirle

$$
\begin{aligned}
& \text { for } \mathrm{i}=1 \text { : } \mathrm{n} \\
& \quad \text { if }\left(\mathrm{K}_{[\mathrm{i}-1]}<\text { konum }<=\mathrm{K}_{[\mathrm{i}]}\right) \\
& \\
& \text { end }
\end{aligned}
$$

\section{B. EFO ALGORITMASI}

EFO [1], elektromıknatıslar arasındaki itme-çekme kuvvetinden esinlenilerek geliştirilmiş topluluk tabanlı bir sezgisel arama algoritmasıdır. Elektromanyetik parçacıklar, optimizasyon problemleri için çözüm adaylarını temsil ederler. Bu parçacıkları oluşturan elektromıknatısların sayısı ise optimizasyon problemlerindeki tasarım değişken sayısına karşılık gelmektedir. EFO algoritmasında, öncelikle diğer MSA algoritmalarında olduğu gibi rastgele bir şekilde çözüm adayları yani elektromıknatıslar üretilir. Çözüm adayları topluluğundaki her bir parçacığın uygunluk değerinin hesabı yapılır. Bunun için probleme ait amaç fonksiyon ve varsa kısıtlar kullanılır. EFO algoritmasında açgözlü seçim yöntemine göre yani çözüm adaylarının uygunluk değerlerine göre üç alt grup oluşturulur. Bunun için öncelikle çözüm adaylarının uygunluk değerlerine bağlı olarak sıralanması gerekir. Uygunluk değerlerine göre sıralanmış parçacıklardan oluşturulan ilk grup pozitif kutba sahip elektromanyetik parçacıkları temsil eder. İkinci grup ise en düşük uygunluğa sahip olan ve kullanıcı tarafindan oranı ve sayısı belirlenen negatif alan parçacıklarıdır. Bu iki gruba dahil edilemeyen parçacıklar ise nötr alanı temsil eden üçüncü grubu oluşturur. Arama operatörleri tarafindan işlenen çözüm adayları (elektromanyetik parçacıklar) ile EFO popülasyonu güncellenir. Güncelleme sürecinde yeni parçacıkların mevcut parçacıklardan daha başarılı bir uygunluk değerine sahip olması şartı gözetilir. EFO algoritmasına ait akış diyagramı Şekil 1 'de verilmektedir. 


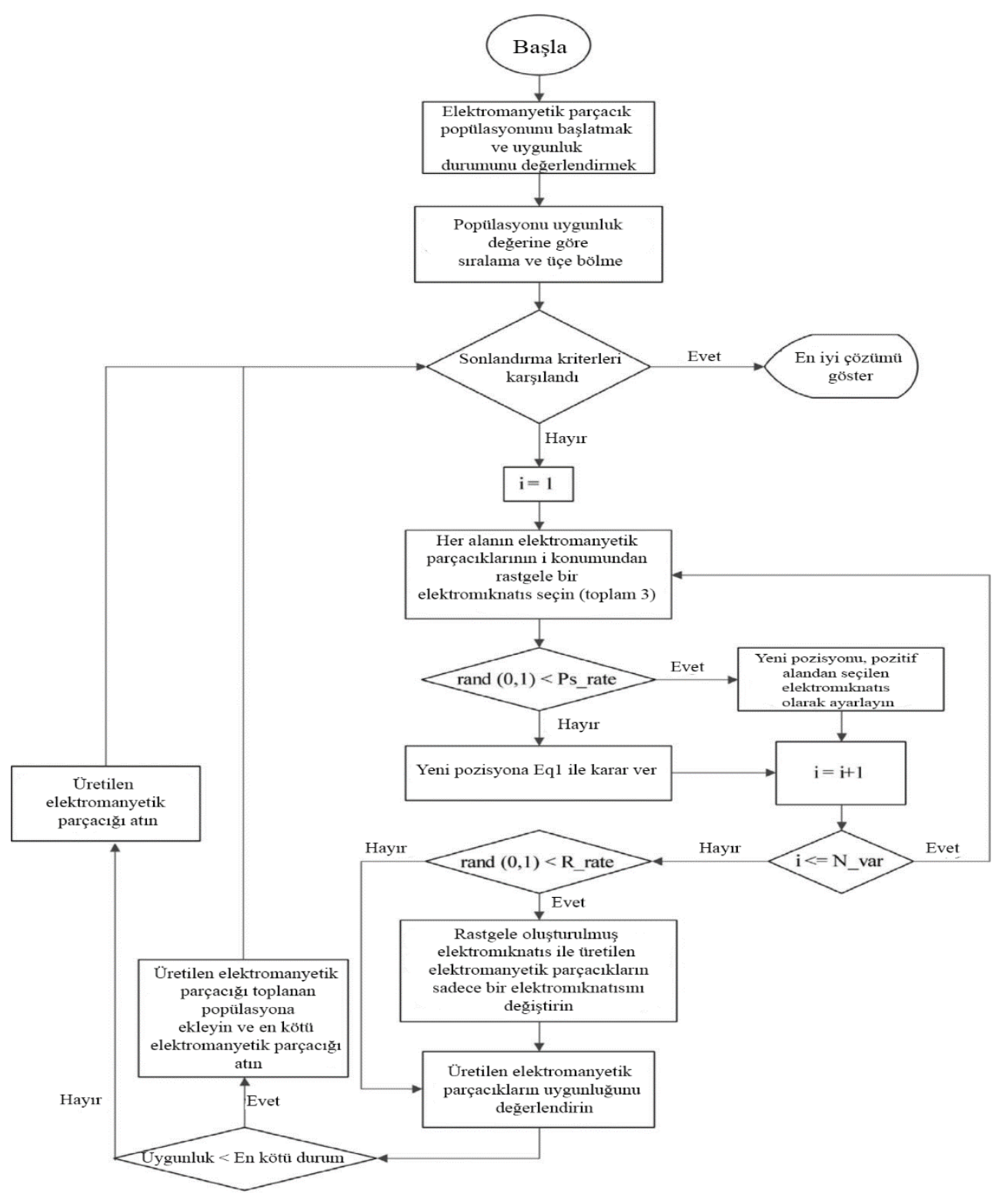

Şekil 1. EFO akış diyagramı [1]

\section{RULET SEÇİM YÖNTEMİ-TABANLI EFO ALGORITMASININ (R-EFO) GELIŞTIRILMESI}

EFO algoritmasının baz modelinde çözüm adayları topluluğu (elektromnanyetik parçacıklar) rasgele yaratıldıktan sonra üç alt popülasyona bölünmektedir. Bunlar doğada olduğu gibi pozitif, nötr ve negatif yüklü parçacıkları temsil etmektedirler. Alt popülasyonlardaki parçacık sayıları daha önceden belirlenen oranlara göre ve parçacıkların uygunluk değerlerine bağlı olarak ayarlanmaktadır. Buna göre parçacıklar uygunluk değerlerine bağlı olarak sıralanmakta ve aç gözlü seçim yöntemi ile alt popülasyonlara dağıtılmaktadırlar. EFO algoritmasında, uygunluk değerlerine bağlı olarak sıralanan popülasyonda ilk $10 \%$ 'da yer alan parçacıklar seçilerek pozitif alanı temsil eden alt popülasyon oluşturulur. Sıralamanın 10\%-55\% arası dilimde yer alan çözüm adayları nötr alanı ve son $45 \%$ ' lik dilimde yer alan parçacıklar ise negatif alanı temsil eden alt popülasyonları oluşturmaktadırlar. Alt popülasyonları oluşturduktan sonra arama süreci yaşam döngüsü başlamaktadır. Bu süreçte ilk olarak elektro mıknatıslar arasındaki itme-çekme kuvvetini temsil eden parametre değeri force $=$ rand $(0,1)$ ifadesi ile hesaplanır. Arama süreci yaşam döngüsü temel olarak üç adımdan oluşmaktadır. Bunlar; seçim işlemleri (arama sürecine rehberlik eden parçacıkların belirlenmesi), komşuluk araması ve çeşitlilik adımlarıdır. Seçim işlemleri 
evresinde EFO popülasyonunu oluşturan üç alandan birer adet parçacık rasgele bir şekilde seçilir. Bunlar pozitif, nötr ve negatif alanlardan rasgele seçilen çözüm adaylarıdır. Önerilen yöntemde de pozitif ve nötr alanlardan parçacık seçim işlemi klasik EFO algoritması ile aynıdır (Algoritma 2'de 25. ve 26. satırlar). Bununla birlikte, önerilen yöntemde negatif alandan parçacık seçim işleminde Rulet yöntemi de kullanılmaktadır. Rulet yönteminin tatbik edilmesine ilişkin sözde-kod Algoritma 2'de 27-31 aras1 satırlarda verilmektedir. Bu satırlardaki sözde kodlardan da anlaşılacağı üzere negatif alandan seçim işlemi problem boyutuna (N_var) bağlı olarak olasılıksal olarak işleyen iki alt adıma bölünmüştür. Problem boyutunun 20\%'sine karşılık gelen bu olasılık "rand $(0,1)<\mathrm{N} \_v a r$ / $(100 * 5)$ " formülü ile hesaplanmaktadır. Buna göre problem boyutunun 20\%'si oranında bir siklıkla Rulet yöntemi kullanılarak popülasyondaki tüm çözüm adayları arasından bir adet parçacık seçilmektedir. Problem boyutunun $80 \%$ 'ine karşılık gelen oranda ise EFO algoritmasının baz modelinde olduğu gibi sadece negatif parçacıklar arasından ve rasgele bir şekilde seçim yapılmaktadır. Seçim işleminden sonra arama sürecinin diğer iki önemli evresi olan komşuluk araması ve çeşitlilik işlemleri gerçekleştirilmektedir. Komşuluk araması görevi, Algoritma 2'de 32-36 satırları arasında verilen işlemlerle ve iki farklı matematiksel ifade ile gerçekleştirilmektedir. Komşuluk araması sürecinde kullanılacak olan yöntemin belirlenmesinde Ps_rate yani pozitif alandan seçilme oranı kullanılmaktadır. $\mathrm{Bu}$ oran EFO algoritmasında $20 \%$ olarak kabul edilmiştir. Pozitif alandan seçilen çözüm adayının komşuluğunda arama yapmak için Algoritma 2'de 33. satırda verilen eşitlik kullanılmaktadır. Pozitif, negatif ve nötr alanlardan seçilen çözüm adaylarının komşuluğunda arama yapmak için ise Algoritma 2'de 35. satırda verilen eşitlik kullanılmaktadır. Arama süreci yaşam döngüsünün son adımı keşif (exploration) sürecidir. Bu sürece ait işlemler Algoritma 2'de 41-43 satırlarında gerçekleştirilmektedir. Bu satırlarda yer alan RI, üzerinde değişiklik yapılan tasarım parametresini temsil etmektedir. Bir başka ifadeyle çözüm adayının (parçacığın) mutasyona uğrayacak olan boyutu RI ile gösterilmektedir. Bu süreçte üretilen çözüm adayının (new_emp) uygunluk değeri ile popülasyondaki en düşük uygunluk değerine sahip olan çözüm adayı karşılaştırması yapılarak popülasyon güncellenir (Algoritma 2'de 48-53 numaralı satırlarda).

\section{Algoritma 2. EFO [1] algoritmasının adımları ve rulet yönteminin uygulanması}

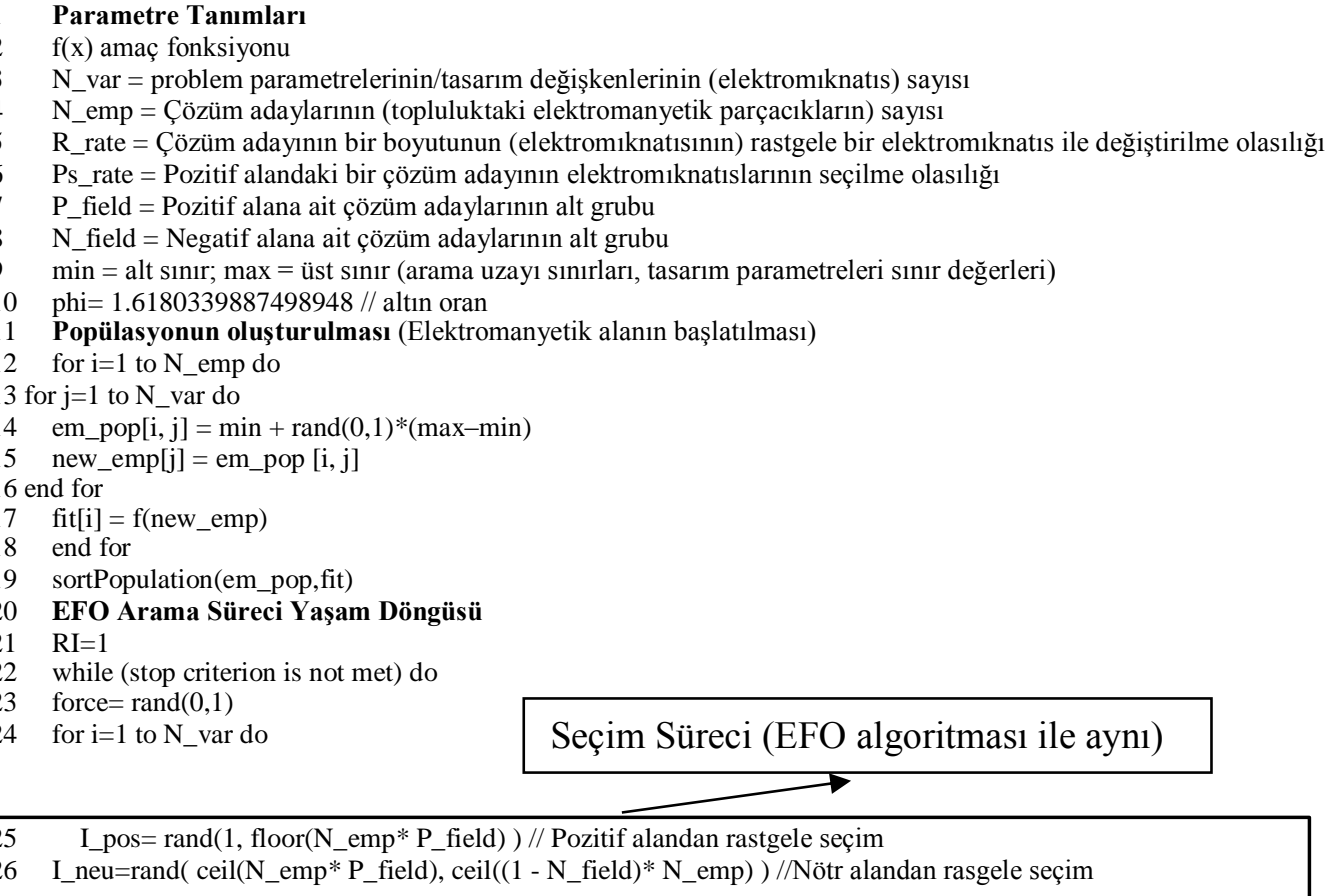

I_pos= rand(1, floor(N_emp* P_field) ) // Pozitif alandan rastgele seçim I_neu=rand ( ceil(N_emp* P_field $)$, ceil $\left(\left(1-N_{-} \text {field }\right)^{*} N_{-}\right.$emp $\left.)\right) / /$Nötr alandan rasgele seçim 


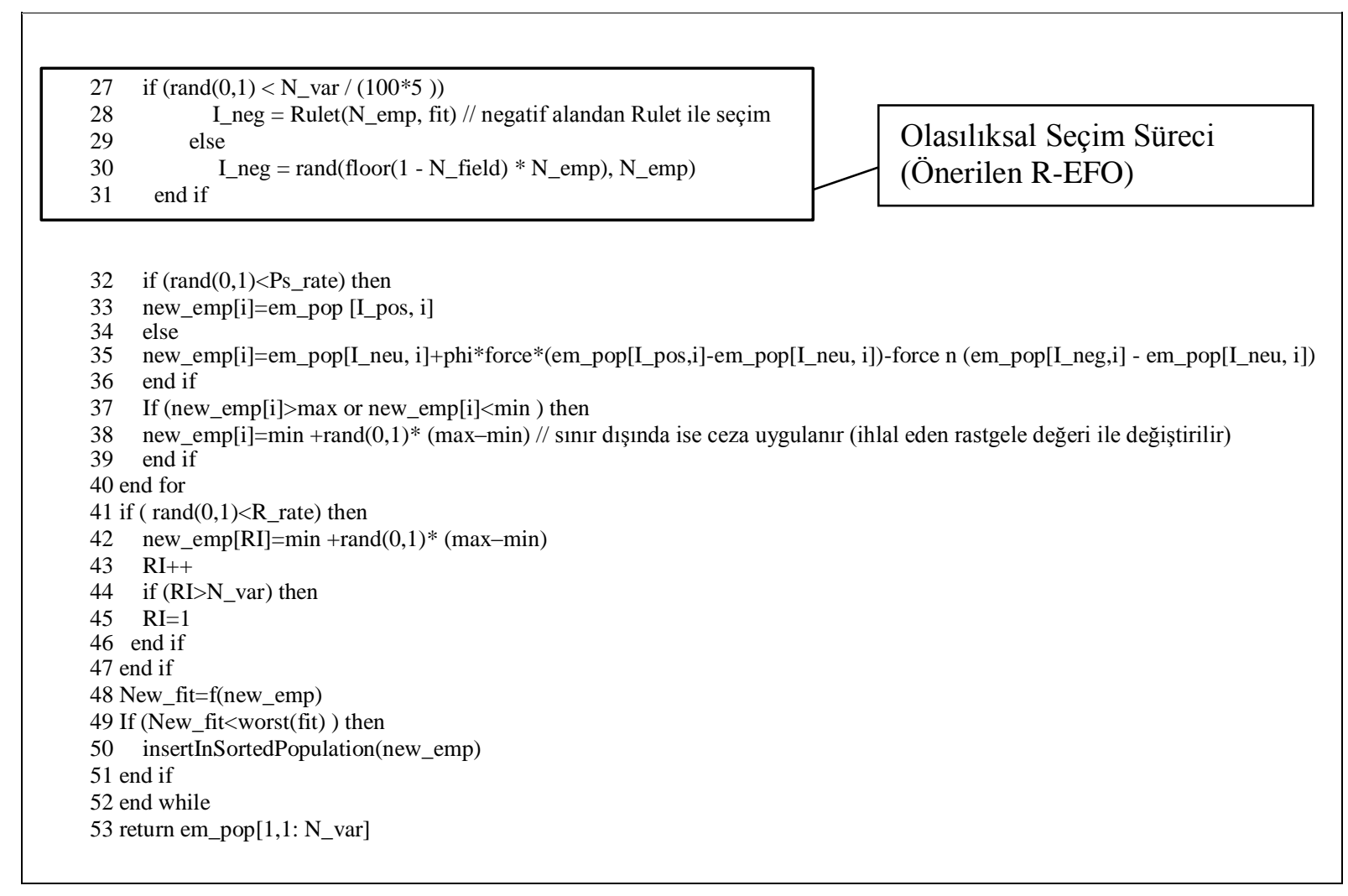

\section{Deneysel Calișma}

\section{A. AYARLAR}

Meta-sezgisel arama algoritmalarının deneysel çalışmalarında standart olarak uygulanması gereken ayarları dört başlık altında toplamak mümkündür. Bunlar; "çalışma tekrar sayısı", "amaç fonksiyonu azami çağırma sayısı ve "karşılaştırma problemleri" ve "problem boyutu" olarak ifade edilebilir. MSA algoritmaları için deneysel çalışmaların standartlarını tanımlama konusunda 1999 yılından buyana CEC konferansları yoluyla katk1 sunulmaktadır [16-17]. Son yıllarda bu konferanslarda MSA algoritmalarının deneysel çalışma tekrar sayısı 51 olarak tanımlanmaktadır. Amaç fonksiyonu azami çağırma sayısı problem boyutunun 10 bin katı olarak belirlenmiştir. 2014 yılından itibaren karşılaştırma problemlerinin bir havuzunun oluşturulduğu ve standart hale geldiği görülmektedir. Havuz, 30 adet sürekli değerli ve dinamik yapılı test problemlerinden oluşmaktadır. Dolayısıyla problemlere ait tasarım parametrelerinin arama uzayındaki optimum konumlarının da kaydırma ve döndürme işlevleri yoluyla (shifted, rotated) dinamik olarak değiştirilebildiği ve bu yolla MSA algoritmalarının çeşitli yollarla optimum noktaları yakalayacak avantajlar yaratmasının önüne geçilmeye çalışıldığ 1 anlaşılmaktadır. Ayrıca MSA algoritmalarının test edilmesinde tercih edilen problem boyutlarının dinamik olmasının gerekliliği dikkate alınarak CEC konferanslarındaki test problemlerinin tamamı dinamik olarak boyutlandırılabilen problemlerle oluşturulmuştur. Böylelikle küçük boyutlu arama uzaylarında hızlı yakınsama özellikleri sayesinde başarılı görülen algoritmaların aynı problemlerin orta ve büyük boyutlu arama uzaylarındaki performanslarını da ortaya çıkarmak amaçlanmıştır. Algoritmaların arama performanslarından kastedilen bilgi sadece tasarım parametrelerinin genel optimum noktalara yakınsama başarısı değildir. Bunun yanında hesaplama karmaşıklığı da algoritma performanslarının ölçülmesinde dikkate alınan bir bilgidir. Özellikle çok boyutlu ve karmaşıklık düzeyi yüksek arama uzaylarında hesaplama karmaşıklığı yüksek algoritmaların kabul edilebilir bir çözüme makul bir hesaplama süresi içerisinde erişmeleri mümkün olmayabilir. Bu bir MSA algoritmasının optimizasyon problemlerinin niteliklerine göre kullanılabilirliğini göstermesi açısından önemli bir bilgidir. $\mathrm{Bu}$ açıklamalara bağlı olarak bu makale çalışmasında önerilen R-EFO algoritmasının deneysel 
çalı̧̧malarında deney tekrar sayısı 51 , amaç fonksiyonu azami çağırma sayısı $10.000 * \mathrm{~d}$ (d, problem boyutu), karşılaştırma problem havuzu CEC 2017 (30 adet sürekli değerli kısıtsız optimizasyon problemi), problem boyutu 30, 50 ve 100 olarak CEC17 konferansındaki şartlar referans alınmıştır [16].

\section{B. DENEYSEL SONUÇLAR}

R-EFO ve EFO algoritmalarının CEC17 test havuzu üzerindeki performansları Wilcoxon yöntemi kullanılarak istatistiksel olarak analiz edilmiştir. Buna göre algoritmaların ikili karşılaştırma sonuçları Tablo 1'in son satırında verilmektedir. Tablo 1'de ayrica test problemlerinin 30, 50 ve 100 boyutları için rakip algoritmaların 30 problem üzerindeki optimizasyon performansları verilmektedir. Buna göre R-EFO algoritması problem boyutu 30 iken EFO algoritmasının baz modeline kiyasla otuz problemin 13'ünde üstünlük kurarken, 16 problemde beraberlik ve 1 problemde de yenilgi elde etmiştir. Problemlerin 50 ve 100 boyutları için elde edilen sonuçlar benzerdir. Bu durum, rulet seçim yönteminin EFO algoritmasına tatbik edilme stratejisinin algoritmanın çalışma karakteristiğine uyum ve katkı sağladığını göstermektedir.

Tablo 1. EFO ve R-EFO algoritmalarının Wilcoxon karşılaştırma sonuçları

\begin{tabular}{|c|c|c|c|c|c|c|c|c|c|c|c|c|c|c|c|c|}
\hline & & \multicolumn{5}{|c|}{ Boyut: 30} & \multicolumn{5}{|c|}{ Boyut: 50} & \multicolumn{5}{|c|}{ Boyut: 100} \\
\hline & & Min & $\begin{array}{c}\text { Ortala } \\
\text { ma }\end{array}$ & Std.S. & $\operatorname{Max}$ & $\mathbf{D}$ & Min & $\begin{array}{c}\text { Ortala } \\
\text { ma }\end{array}$ & Std.S. & Max & D & Min & $\begin{array}{c}\text { Ortala } \\
\text { ma }\end{array}$ & Std.S. & Max & D \\
\hline \multirow{2}{*}{1} & EFO & $\begin{array}{c}5,09 \mathrm{E}- \\
04\end{array}$ & $\begin{array}{c}3,15 \mathrm{E}+ \\
03\end{array}$ & $\begin{array}{c}3,64 \mathrm{E} \\
+03\end{array}$ & $\begin{array}{c}1,63 \mathrm{E} \\
+04\end{array}$ & & $\begin{array}{c}6,80 \mathrm{E}- \\
01\end{array}$ & $\begin{array}{c}3,71 \mathrm{E}+ \\
03\end{array}$ & $\begin{array}{c}3,87 \mathrm{E} \\
+03\end{array}$ & $\begin{array}{c}1,41 \mathrm{E} \\
+04\end{array}$ & & $\begin{array}{c}9,26 \mathrm{E} \\
+00\end{array}$ & $\begin{array}{c}7,86 \mathrm{E}+ \\
03\end{array}$ & $\begin{array}{c}1,08 \mathrm{E}+ \\
04\end{array}$ & $\begin{array}{c}3,95 \mathrm{E}+ \\
04\end{array}$ & \\
\hline & $\begin{array}{c}\text { R- } \\
\text { EFO }\end{array}$ & $\begin{array}{c}1,76 \mathrm{E} \\
+01\end{array}$ & $\begin{array}{c}3,03 \mathrm{E}+ \\
03\end{array}$ & $\begin{array}{c}3,78 \mathrm{E} \\
+03\end{array}$ & $\begin{array}{c}1,72 \mathrm{E} \\
+04\end{array}$ & $=$ & $\begin{array}{c}2,15 \mathrm{E} \\
+01\end{array}$ & $\begin{array}{c}3,48 \mathrm{E}+ \\
03\end{array}$ & $\begin{array}{c}3,49 \mathrm{E} \\
+03\end{array}$ & $\begin{array}{c}1,53 \mathrm{E} \\
+04\end{array}$ & $=$ & $\begin{array}{c}3,42 \mathrm{E} \\
+00\end{array}$ & $\begin{array}{c}7,38 \mathrm{E}+ \\
03\end{array}$ & $\begin{array}{c}7,76 \mathrm{E}+ \\
03\end{array}$ & $\begin{array}{c}3,54 \mathrm{E}+ \\
04\end{array}$ & $=$ \\
\hline \multirow{2}{*}{2} & EFO & $\begin{array}{c}8,14 \mathrm{E} \\
+02\end{array}$ & $\begin{array}{c}2,53 \mathrm{E}+ \\
18\end{array}$ & $\begin{array}{c}1,81 \mathrm{E} \\
+19\end{array}$ & $\begin{array}{c}1,29 \mathrm{E} \\
+20\end{array}$ & & $\begin{array}{c}7,23 \mathrm{E} \\
+13\end{array}$ & $\begin{array}{c}1,72 \mathrm{E}+ \\
41\end{array}$ & $\begin{array}{c}1,23 \mathrm{E} \\
+42\end{array}$ & $\begin{array}{c}8,77 \mathrm{E} \\
+42\end{array}$ & & $\begin{array}{c}1,05 \mathrm{E} \\
+71\end{array}$ & $\begin{array}{c}5,20 \mathrm{E}+ \\
115\end{array}$ & $\begin{array}{c}3,71 \mathrm{E}+ \\
116\end{array}$ & $\begin{array}{c}2,65 \mathrm{E}+ \\
117\end{array}$ & \\
\hline & $\begin{array}{c}\text { R- } \\
\text { EFO }\end{array}$ & $\begin{array}{c}0,00 \mathrm{E} \\
+00\end{array}$ & $\begin{array}{c}7,52 \mathrm{E}+ \\
10\end{array}$ & $\begin{array}{c}5,28 \mathrm{E} \\
+11 \\
\end{array}$ & $\begin{array}{c}3,77 \mathrm{E} \\
+12 \\
\end{array}$ & + & $\begin{array}{c}3,00 \mathrm{E} \\
+00\end{array}$ & $\begin{array}{c}9,64 \mathrm{E}+ \\
24\end{array}$ & $\begin{array}{c}6,88 \mathrm{E} \\
+25 \\
\end{array}$ & $\begin{array}{c}4,91 \mathrm{E} \\
+26\end{array}$ & + & $\begin{array}{c}7,35 \mathrm{E} \\
+21 \\
\end{array}$ & $\begin{array}{c}8,71 \mathrm{E}+ \\
90\end{array}$ & $\begin{array}{c}6,22 \mathrm{E}+ \\
91\end{array}$ & $\begin{array}{c}4,44 \mathrm{E}+ \\
92\end{array}$ & + \\
\hline \multirow[b]{2}{*}{3} & EFO & $\begin{array}{c}5,68 \mathrm{E}- \\
14 \\
\end{array}$ & $\begin{array}{c}1,06 \mathrm{E}- \\
12 \\
\end{array}$ & $\begin{array}{c}4,81 \mathrm{E}- \\
12 \\
\end{array}$ & $\begin{array}{c}3,38 \mathrm{E}- \\
11 \\
\end{array}$ & & $\begin{array}{c}8,64 \mathrm{E}- \\
12 \\
\end{array}$ & $\begin{array}{c}2,76 \mathrm{E}- \\
07 \\
\end{array}$ & $\begin{array}{c}1,01 \mathrm{E}- \\
06\end{array}$ & $\begin{array}{c}5,32 \mathrm{E}- \\
06 \\
\end{array}$ & & $\begin{array}{c}3,91 \mathrm{E} \\
+02 \\
\end{array}$ & $\begin{array}{c}8,61 \mathrm{E}+ \\
03 \\
\end{array}$ & $\begin{array}{c}6,22 \mathrm{E}+ \\
03 \\
\end{array}$ & $\begin{array}{c}2,86 \mathrm{E}+ \\
04 \\
\end{array}$ & \\
\hline & $\begin{array}{c}\text { R- } \\
\text { EFO }\end{array}$ & $\begin{array}{c}5,68 \mathrm{E}- \\
14\end{array}$ & $\begin{array}{c}1,95 \mathrm{E}- \\
13\end{array}$ & $\begin{array}{c}1,40 \mathrm{E}- \\
13\end{array}$ & $\begin{array}{c}7,96 \mathrm{E}- \\
13\end{array}$ & $=$ & $\begin{array}{c}3,33 \mathrm{E}- \\
06\end{array}$ & $\begin{array}{c}4,49 \mathrm{E}- \\
02\end{array}$ & $\begin{array}{c}1,55 \mathrm{E}- \\
01\end{array}$ & $\begin{array}{c}8,85 \mathrm{E}- \\
01\end{array}$ & - & $\begin{array}{c}3,64 \mathrm{E} \\
+03\end{array}$ & $\begin{array}{c}1,11 \mathrm{E}+ \\
04\end{array}$ & $\begin{array}{c}4,37 \mathrm{E}+ \\
03\end{array}$ & $\begin{array}{c}2,51 \mathrm{E}+ \\
04\end{array}$ & - \\
\hline \multirow{2}{*}{4} & EFO & $\begin{array}{c}5,84 \mathrm{E}- \\
04\end{array}$ & $\begin{array}{c}1,14 \mathrm{E}+ \\
01\end{array}$ & $\begin{array}{c}2,23 \mathrm{E} \\
+01\end{array}$ & $\begin{array}{c}7,19 \mathrm{E} \\
+01\end{array}$ & & $\begin{array}{c}5,76 \mathrm{E}- \\
06\end{array}$ & $\begin{array}{c}3,49 \mathrm{E}+ \\
01\end{array}$ & $\begin{array}{c}4,51 \mathrm{E} \\
+01\end{array}$ & $\begin{array}{c}1,48 \mathrm{E} \\
+02\end{array}$ & & $\begin{array}{c}6,33 \mathrm{E}- \\
05\end{array}$ & $\begin{array}{c}9,06 \mathrm{E}+ \\
01\end{array}$ & $\begin{array}{c}5,71 \mathrm{E}+ \\
01\end{array}$ & $\begin{array}{c}2,18 \mathrm{E}+ \\
02\end{array}$ & \\
\hline & $\begin{array}{c}\text { R- } \\
\text { EFO }\end{array}$ & $\begin{array}{c}, 33 \mathrm{E}- \\
04 \\
\end{array}$ & $\begin{array}{c}1,27 \mathrm{E}+ \\
01\end{array}$ & $\begin{array}{c}2,31 \mathrm{E} \\
+01 \\
\end{array}$ & $\begin{array}{c}7,19 \mathrm{E} \\
+01 \\
\end{array}$ & $=$ & $\begin{array}{c}1,84 \mathrm{E}- \\
04 \\
\end{array}$ & $\begin{array}{c}3,85 \mathrm{E}+ \\
01\end{array}$ & $\begin{array}{c}4,54 \mathrm{E} \\
+01 \\
\end{array}$ & $\begin{array}{c}1,18 \mathrm{E} \\
+02\end{array}$ & $=$ & $\begin{array}{c}7,00 \mathrm{E} \\
+00 \\
\end{array}$ & $\begin{array}{c}1,26 \mathrm{E}+ \\
02\end{array}$ & $\begin{array}{c}5,91 \mathrm{E}+ \\
01\end{array}$ & $\begin{array}{c}2,55 \mathrm{E}+ \\
02\end{array}$ & - \\
\hline \multirow[b]{2}{*}{5} & EFO & $\begin{array}{c}1,29 \mathrm{E} \\
+01\end{array}$ & $\begin{array}{c}3,24 \mathrm{E}+ \\
01\end{array}$ & $\begin{array}{c}8,89 \mathrm{E} \\
+00 \\
\end{array}$ & $\begin{array}{c}5,37 \mathrm{E} \\
+01 \\
\end{array}$ & & $\begin{array}{c}3,98 \mathrm{E} \\
+01\end{array}$ & $\begin{array}{c}7,82 \mathrm{E}+ \\
01\end{array}$ & $\begin{array}{c}1,91 \mathrm{E} \\
+01\end{array}$ & $\begin{array}{c}1,27 \mathrm{E} \\
+02\end{array}$ & & $\begin{array}{c}1,80 \mathrm{E} \\
+02\end{array}$ & $\begin{array}{c}2,51 \mathrm{E}+ \\
02\end{array}$ & $\begin{array}{c}3,77 \mathrm{E}+ \\
01\end{array}$ & $\begin{array}{c}3,48 \mathrm{E}+ \\
02\end{array}$ & \\
\hline & $\begin{array}{c}\text { R- } \\
\text { EFO }\end{array}$ & $\begin{array}{c}1,19 \mathrm{E} \\
+01\end{array}$ & $\begin{array}{c}2,43 \mathrm{E}+ \\
01\end{array}$ & $\begin{array}{c}6,00 \mathrm{E} \\
+00\end{array}$ & $\begin{array}{c}3,68 \mathrm{E} \\
+01\end{array}$ & + & $\begin{array}{c}3,38 \mathrm{E} \\
+01\end{array}$ & $\begin{array}{c}6,29 \mathrm{E}+ \\
01\end{array}$ & $\begin{array}{c}1,15 \mathrm{E} \\
+01\end{array}$ & $\begin{array}{c}8,46 \mathrm{E} \\
+01\end{array}$ & + & $\begin{array}{c}1,66 \mathrm{E} \\
+02\end{array}$ & $\begin{array}{c}2,27 \mathrm{E}+ \\
02\end{array}$ & $\begin{array}{c}3,44 \mathrm{E}+ \\
01\end{array}$ & $\begin{array}{c}2,96 \mathrm{E}+ \\
02\end{array}$ & + \\
\hline \multirow[b]{2}{*}{6} & EFO & $\begin{array}{c}3,95 \mathrm{E}- \\
03\end{array}$ & $\begin{array}{c}2,85 \mathrm{E}- \\
01\end{array}$ & $\begin{array}{c}3,14 \mathrm{E}- \\
01\end{array}$ & $\begin{array}{c}1,41 \mathrm{E} \\
+00\end{array}$ & & $\begin{array}{c}3,55 \mathrm{E}- \\
01\end{array}$ & $\begin{array}{c}2,20 \mathrm{E}+ \\
00\end{array}$ & $\begin{array}{c}1,25 \mathrm{E} \\
+00\end{array}$ & $\begin{array}{c}5,72 \mathrm{E} \\
+00\end{array}$ & & $\begin{array}{c}7,46 \mathrm{E} \\
+00\end{array}$ & $\begin{array}{c}1,22 \mathrm{E}+ \\
01\end{array}$ & $\begin{array}{c}2,33 \mathrm{E}+ \\
00\end{array}$ & $\begin{array}{c}1,80 \mathrm{E}+ \\
01\end{array}$ & \\
\hline & $\begin{array}{c}\text { R- } \\
\text { EFO }\end{array}$ & $\begin{array}{c}6,05 \mathrm{E}- \\
05\end{array}$ & $\begin{array}{c}2,48 \mathrm{E}- \\
03\end{array}$ & $\begin{array}{c}2,69 \mathrm{E}- \\
03\end{array}$ & $\begin{array}{c}1,30 \mathrm{E}- \\
02 \\
\end{array}$ & + & $\begin{array}{c}1,17 \mathrm{E}- \\
03 \\
\end{array}$ & $\begin{array}{c}7,86 \mathrm{E}- \\
03\end{array}$ & $\begin{array}{c}5,02 \mathrm{E}- \\
03 \\
\end{array}$ & $\begin{array}{c}1,97 \mathrm{E}- \\
02 \\
\end{array}$ & + & $\begin{array}{c}6,85 \mathrm{E}- \\
03 \\
\end{array}$ & $\begin{array}{c}2,54 \mathrm{E}- \\
02\end{array}$ & $\begin{array}{c}1,15 \mathrm{E}- \\
02\end{array}$ & $\begin{array}{c}5,52 \mathrm{E}- \\
02\end{array}$ & + \\
\hline \multirow{2}{*}{7} & EFO & $\begin{array}{c}4,75 \mathrm{E} \\
+01 \\
\end{array}$ & $\begin{array}{c}7,17 \mathrm{E}+ \\
01\end{array}$ & $\begin{array}{c}2,21 \mathrm{E} \\
+01\end{array}$ & $\begin{array}{c}1,67 \mathrm{E} \\
+02 \\
\end{array}$ & & $\begin{array}{c}1,06 \mathrm{E} \\
+02 \\
\end{array}$ & $\begin{array}{c}1,67 \mathrm{E}+ \\
02\end{array}$ & $\begin{array}{c}3,18 \mathrm{E} \\
+01 \\
\end{array}$ & $\begin{array}{c}2,53 \mathrm{E} \\
+02 \\
\end{array}$ & & $\begin{array}{c}4,72 \mathrm{E} \\
+02 \\
\end{array}$ & $\begin{array}{c}7,49 \mathrm{E}+ \\
02\end{array}$ & $\begin{array}{c}1,53 \mathrm{E}+ \\
02\end{array}$ & $\begin{array}{c}1,15 \mathrm{E}+ \\
03\end{array}$ & \\
\hline & $\begin{array}{c}\text { R- } \\
\text { EFO }\end{array}$ & $\begin{array}{c}4,51 \mathrm{E} \\
+01\end{array}$ & $\begin{array}{c}7,29 \mathrm{E}+ \\
01\end{array}$ & $\begin{array}{c}3,80 \mathrm{E} \\
+01\end{array}$ & $\begin{array}{c}1,86 \mathrm{E} \\
+02 \\
\end{array}$ & + & $\begin{array}{c}9,24 \mathrm{E} \\
+01\end{array}$ & $\begin{array}{c}1,32 \mathrm{E}+ \\
02\end{array}$ & $\begin{array}{c}4,85 \mathrm{E} \\
+01 \\
\end{array}$ & $\begin{array}{c}4,48 \mathrm{E} \\
+02\end{array}$ & + & $\begin{array}{c}2,93 \mathrm{E} \\
+02\end{array}$ & $\begin{array}{c}4,42 \mathrm{E}+ \\
02\end{array}$ & $\begin{array}{c}\text { 5,34E+ } \\
01\end{array}$ & $\begin{array}{c}5,40 \mathrm{E}+ \\
02\end{array}$ & + \\
\hline \multirow{2}{*}{8} & EFO & $\begin{array}{c}1,49 \mathrm{E} \\
+01\end{array}$ & $\begin{array}{c}3,21 \mathrm{E}+ \\
01\end{array}$ & $\begin{array}{c}1,07 \mathrm{E} \\
+01 \\
\end{array}$ & $\begin{array}{c}6,47 \mathrm{E} \\
+01 \\
\end{array}$ & & $\begin{array}{c}4,08 \mathrm{E} \\
+01 \\
\end{array}$ & $\begin{array}{c}7,39 \mathrm{E}+ \\
01\end{array}$ & $\begin{array}{c}1,82 \mathrm{E} \\
+01 \\
\end{array}$ & $\begin{array}{c}1,21 \mathrm{E} \\
+02\end{array}$ & & $\begin{array}{c}1,85 \mathrm{E} \\
+02 \\
\end{array}$ & $\begin{array}{c}2,67 \mathrm{E}+ \\
02\end{array}$ & $\begin{array}{c}4,03 \mathrm{E}+ \\
01\end{array}$ & $\begin{array}{c}3,87 \mathrm{E}+ \\
02\end{array}$ & \\
\hline & $\begin{array}{c}\text { R- } \\
\text { EFO }\end{array}$ & $\begin{array}{c}1,39 \mathrm{E} \\
+01\end{array}$ & $\begin{array}{c}2,49 \mathrm{E}+ \\
01\end{array}$ & $\begin{array}{c}5,64 \mathrm{E} \\
+00\end{array}$ & $\begin{array}{c}4,08 \mathrm{E} \\
+01\end{array}$ & + & $\begin{array}{c}3,18 \mathrm{E} \\
+01\end{array}$ & $\begin{array}{c}5,73 \mathrm{E}+ \\
01\end{array}$ & $\begin{array}{c}1,19 \mathrm{E} \\
+01\end{array}$ & $\begin{array}{c}8,46 \mathrm{E} \\
+01\end{array}$ & + & $\begin{array}{c}1,59 \mathrm{E} \\
+02\end{array}$ & $\begin{array}{c}2,27 \mathrm{E}+ \\
02\end{array}$ & $\begin{array}{c}3,26 \mathrm{E}+ \\
01\end{array}$ & $\begin{array}{c}3,36 \mathrm{E}+ \\
02\end{array}$ & + \\
\hline \multirow{2}{*}{9} & EFO & $\begin{array}{c}7,23 \mathrm{E}- \\
01\end{array}$ & $\begin{array}{c}3,94 \mathrm{E}+ \\
01\end{array}$ & $\begin{array}{c}5,52 \mathrm{E} \\
+01\end{array}$ & $\begin{array}{c}3,07 \mathrm{E} \\
+02\end{array}$ & & $\begin{array}{c}1,04 \mathrm{E} \\
+02\end{array}$ & $\begin{array}{c}4,98 \mathrm{E}+ \\
02\end{array}$ & $\begin{array}{c}4,03 \mathrm{E} \\
+02\end{array}$ & $\begin{array}{c}1,97 \mathrm{E} \\
+03\end{array}$ & & $\begin{array}{c}2,01 \mathrm{E} \\
+03\end{array}$ & $\begin{array}{c}1,23 \mathrm{E}+ \\
04\end{array}$ & $\begin{array}{c}4,24 \mathrm{E}+ \\
03\end{array}$ & $\begin{array}{c}2,12 \mathrm{E}+ \\
04\end{array}$ & \\
\hline & $\begin{array}{c}\text { R- } \\
\text { EFO }\end{array}$ & $\begin{array}{c}5,44 \mathrm{E}- \\
01 \\
\end{array}$ & $\begin{array}{c}1,19 \mathrm{E}+ \\
01\end{array}$ & $\begin{array}{c}9,83 \mathrm{E} \\
+00\end{array}$ & $\begin{array}{c}4,30 \mathrm{E} \\
+01 \\
\end{array}$ & + & $\begin{array}{c}3,26 \mathrm{E} \\
+01 \\
\end{array}$ & $\begin{array}{c}1,31 \mathrm{E}+ \\
02\end{array}$ & $\begin{array}{c}6,84 \mathrm{E} \\
+01 \\
\end{array}$ & $\begin{array}{c}3,06 \mathrm{E} \\
+02 \\
\end{array}$ & + & $\begin{array}{c}7,02 \mathrm{E} \\
+02 \\
\end{array}$ & $\begin{array}{c}2,19 \mathrm{E}+ \\
03\end{array}$ & $\begin{array}{c}2,36 \mathrm{E}+ \\
03\end{array}$ & $\begin{array}{c}1,49 \mathrm{E}+ \\
04\end{array}$ & + \\
\hline \multirow{2}{*}{10} & EFO & $\begin{array}{c}7,34 \mathrm{E} \\
+02 \\
\end{array}$ & $\begin{array}{c}5,74 \mathrm{E}+ \\
03 \\
\end{array}$ & $\begin{array}{c}1,80 \mathrm{E} \\
+03 \\
\end{array}$ & $\begin{array}{c}7,29 \mathrm{E} \\
+03 \\
\end{array}$ & & $\begin{array}{c}1,39 \mathrm{E} \\
+03 \\
\end{array}$ & $\begin{array}{c}1,23 \mathrm{E}+ \\
04\end{array}$ & $\begin{array}{c}1,65 \mathrm{E} \\
+03 \\
\end{array}$ & $\begin{array}{c}1,34 \mathrm{E} \\
+04 \\
\end{array}$ & & $\begin{array}{c}2,73 \mathrm{E} \\
+04 \\
\end{array}$ & $\begin{array}{c}2,92 \mathrm{E}+ \\
04\end{array}$ & $\begin{array}{c}6,22 \mathrm{E}+ \\
02 \\
\end{array}$ & $\begin{array}{c}3,02 \mathrm{E}+ \\
04\end{array}$ & \\
\hline & $\begin{array}{c}\text { R- } \\
\text { EFO }\end{array}$ & $\begin{array}{c}1,34 \mathrm{E} \\
+02 \\
\end{array}$ & $\begin{array}{c}5,80 \mathrm{E}+ \\
03 \\
\end{array}$ & $\begin{array}{c}1,80 \mathrm{E} \\
+03 \\
\end{array}$ & $\begin{array}{c}7,33 \mathrm{E} \\
+03 \\
\end{array}$ & $=$ & $\begin{array}{c}6,07 \mathrm{E} \\
+03 \\
\end{array}$ & $\begin{array}{c}1,25 \mathrm{E}+ \\
04\end{array}$ & $\begin{array}{c}1,02 \mathrm{E} \\
+03 \\
\end{array}$ & $\begin{array}{c}1,37 \mathrm{E} \\
+04 \\
\end{array}$ & $=$ & $\begin{array}{c}2,75 \mathrm{E} \\
+04 \\
\end{array}$ & $\begin{array}{c}2,93 \mathrm{E}+ \\
04\end{array}$ & $\begin{array}{c}5,56 \mathrm{E}+ \\
02 \\
\end{array}$ & $\begin{array}{c}3,05 \mathrm{E}+ \\
04\end{array}$ & $=$ \\
\hline \multirow{2}{*}{11} & EFO & $\begin{array}{c}5,97 \mathrm{E} \\
+00\end{array}$ & $\begin{array}{c}3,55 \mathrm{E}+ \\
01\end{array}$ & $\begin{array}{c}2,72 \mathrm{E} \\
+01\end{array}$ & $\begin{array}{c}1,12 \mathrm{E} \\
+02\end{array}$ & & $\begin{array}{c}2,39 \mathrm{E} \\
+01\end{array}$ & $\begin{array}{c}8,19 \mathrm{E}+ \\
01\end{array}$ & $\begin{array}{c}2,95 \mathrm{E} \\
+01\end{array}$ & $\begin{array}{c}1,63 \mathrm{E} \\
+02\end{array}$ & & $\begin{array}{c}2,04 \mathrm{E} \\
+02\end{array}$ & $\begin{array}{c}3,82 \mathrm{E}+ \\
02\end{array}$ & $\begin{array}{c}1,09 \mathrm{E}+ \\
02\end{array}$ & $\begin{array}{c}6,56 \mathrm{E}+ \\
02\end{array}$ & \\
\hline & $\begin{array}{c}\text { R- } \\
\text { EFO }\end{array}$ & $\begin{array}{c}3,98 \mathrm{E} \\
+00 \\
\end{array}$ & $\begin{array}{c}2,94 \mathrm{E}+ \\
01\end{array}$ & $\begin{array}{c}3,07 \mathrm{E} \\
+01\end{array}$ & $\begin{array}{c}1,47 \mathrm{E} \\
+02 \\
\end{array}$ & + & $\begin{array}{c}1,60 \mathrm{E} \\
+01 \\
\end{array}$ & $\begin{array}{c}5,92 \mathrm{E}+ \\
01\end{array}$ & $\begin{array}{c}8,58 \mathrm{E} \\
+01 \\
\end{array}$ & $\begin{array}{c}5,39 \mathrm{E} \\
+02 \\
\end{array}$ & + & $\begin{array}{c}4,75 \mathrm{E} \\
+01 \\
\end{array}$ & $\begin{array}{c}1,33 \mathrm{E}+ \\
02\end{array}$ & $\begin{array}{c}5,56 \mathrm{E}+ \\
01\end{array}$ & $\begin{array}{c}2,90 \mathrm{E}+ \\
02\end{array}$ & + \\
\hline
\end{tabular}


Tablo 1 (devam). EFO ve R-EFO algoritmalarının Wilcoxon karşılaştırma sonuçları

\begin{tabular}{|c|c|c|c|c|c|c|c|c|c|c|c|c|c|c|c|c|}
\hline \multirow{2}{*}{12} & EFO & $\begin{array}{c}1,99 \mathrm{E}+ \\
03\end{array}$ & $\begin{array}{c}1,50 \mathrm{E}+ \\
04\end{array}$ & $\begin{array}{c}8,89 \mathrm{E}+ \\
03\end{array}$ & $\begin{array}{c}3,74 \mathrm{E}+ \\
04\end{array}$ & & $\begin{array}{c}4,08 \mathrm{E}+ \\
03\end{array}$ & $\begin{array}{c}5,12 \mathrm{E}+ \\
04\end{array}$ & $\begin{array}{c}3,31 \mathrm{E}+ \\
04\end{array}$ & $\begin{array}{c}1,55 \mathrm{E}+ \\
05\end{array}$ & & $\begin{array}{c}5,84 \mathrm{E}+ \\
04\end{array}$ & $\begin{array}{c}2,12 \mathrm{E}+ \\
05\end{array}$ & $\begin{array}{c}3,62 \mathrm{E}+ \\
05\end{array}$ & $\begin{array}{c}2,71 \mathrm{E}+ \\
06\end{array}$ & \\
\hline & $\begin{array}{c}\text { R- } \\
\text { EFO }\end{array}$ & $\begin{array}{c}2,89 \mathrm{E}+ \\
03\end{array}$ & $\begin{array}{c}2,19 \mathrm{E}+ \\
04\end{array}$ & $\begin{array}{c}3,51 \mathrm{E}+ \\
04\end{array}$ & $\begin{array}{c}2,57 \mathrm{E}+ \\
05\end{array}$ & $=$ & $\begin{array}{c}2,46 \mathrm{E}+ \\
04\end{array}$ & $\begin{array}{c}3,80 \mathrm{E}+ \\
05\end{array}$ & $\begin{array}{c}4,81 \mathrm{E}+ \\
05\end{array}$ & $\begin{array}{c}1,57 \mathrm{E}+ \\
06\end{array}$ & - & $\begin{array}{c}1,71 \mathrm{E}+ \\
05\end{array}$ & $\begin{array}{c}\text { 7,37E+ } \\
05\end{array}$ & $\begin{array}{c}9,28 \mathrm{E}+ \\
05\end{array}$ & $\begin{array}{c}3,76 \mathrm{E}+ \\
06\end{array}$ & \\
\hline \multirow{2}{*}{13} & EFO & $\begin{array}{c}9,73 \mathrm{E}+ \\
01\end{array}$ & $\begin{array}{c}1,12 \mathrm{E}+ \\
04\end{array}$ & $\begin{array}{c}1,21 \mathrm{E}+ \\
04\end{array}$ & $\begin{array}{c}4,57 \mathrm{E}+ \\
04\end{array}$ & & $\begin{array}{c}1,28 \mathrm{E}+ \\
02\end{array}$ & $\begin{array}{c}6,21 \mathrm{E}+ \\
03\end{array}$ & $\begin{array}{c}6,18 \mathrm{E}+ \\
03\end{array}$ & $\begin{array}{c}2,17 \mathrm{E}+ \\
04\end{array}$ & & $\begin{array}{c}3,76 \mathrm{E}+ \\
02\end{array}$ & $\begin{array}{c}5,91 \mathrm{E}+ \\
03\end{array}$ & $\begin{array}{c}5,39 \mathrm{E}+ \\
03\end{array}$ & $\begin{array}{c}2,92 \mathrm{E}+ \\
04\end{array}$ & \\
\hline & $\begin{array}{c}\text { R- } \\
\text { EFO }\end{array}$ & $\begin{array}{c}4,82 \mathrm{E}+ \\
01\end{array}$ & $\begin{array}{c}1,09 \mathrm{E}+ \\
04\end{array}$ & $\begin{array}{c}1,08 \mathrm{E}+ \\
04\end{array}$ & $\begin{array}{c}4,25 \mathrm{E}+ \\
04\end{array}$ & $=$ & $\begin{array}{c}4,92 \mathrm{E}+ \\
01\end{array}$ & $\begin{array}{c}3,24 \mathrm{E}+ \\
03\end{array}$ & $\begin{array}{c}4,32 \mathrm{E}+ \\
03\end{array}$ & $\begin{array}{c}1,63 \mathrm{E}+ \\
04\end{array}$ & + & $\begin{array}{c}7,43 \mathrm{E}+ \\
01\end{array}$ & $\begin{array}{c}3,24 \mathrm{E}+ \\
03\end{array}$ & $\begin{array}{c}2,96 \mathrm{E}+ \\
03\end{array}$ & $\begin{array}{c}1,21 \mathrm{E}+ \\
04\end{array}$ & + \\
\hline \multirow{2}{*}{14} & EFO & $\begin{array}{c}1,74 \mathrm{E}+ \\
01\end{array}$ & $\begin{array}{c}2,05 \mathrm{E}+ \\
03\end{array}$ & $\begin{array}{c}2,28 \mathrm{E}+ \\
03\end{array}$ & $\begin{array}{c}1,13 \mathrm{E}+ \\
04\end{array}$ & & $\begin{array}{c}4,71 \mathrm{E}+ \\
02\end{array}$ & $\begin{array}{c}1,05 \mathrm{E}+ \\
04\end{array}$ & $\begin{array}{c}1,50 \mathrm{E}+ \\
04\end{array}$ & $\begin{array}{c}8,48 \mathrm{E}+ \\
04\end{array}$ & & $\begin{array}{c}6,51 \mathrm{E}+ \\
03\end{array}$ & $\begin{array}{c}3,24 \mathrm{E}+ \\
04\end{array}$ & $\begin{array}{c}2,52 \mathrm{E}+ \\
04\end{array}$ & $\begin{array}{c}1,57 \mathrm{E}+ \\
05\end{array}$ & \\
\hline & $\begin{array}{c}\text { R- } \\
\text { EFO }\end{array}$ & $\begin{array}{c}1,35 \mathrm{E}+ \\
02\end{array}$ & $\begin{array}{c}1,06 \mathrm{E}+ \\
04\end{array}$ & $\begin{array}{c}8,94 \mathrm{E}+ \\
03\end{array}$ & $\begin{array}{c}3,53 \mathrm{E}+ \\
04\end{array}$ & - & $\begin{array}{c}2,97 \mathrm{E}+ \\
03\end{array}$ & $\begin{array}{c}3,64 \mathrm{E}+ \\
04\end{array}$ & $\begin{array}{c}3,22 \mathrm{E}+ \\
04\end{array}$ & $\begin{array}{c}1,30 \mathrm{E}+ \\
05\end{array}$ & - & $\begin{array}{c}1,76 \mathrm{E}+ \\
04\end{array}$ & $\begin{array}{c}1,55 \mathrm{E}+ \\
05\end{array}$ & $\begin{array}{c}8,91 \mathrm{E}+ \\
04\end{array}$ & $\begin{array}{c}3,24 \mathrm{E}+ \\
05\end{array}$ & - \\
\hline \multirow{2}{*}{15} & EFO & $\begin{array}{c}2,94 \mathrm{E}+ \\
01\end{array}$ & $\begin{array}{c}5,82 \mathrm{E}+ \\
03\end{array}$ & $\begin{array}{c}6,97 \mathrm{E}+ \\
03\end{array}$ & $\begin{array}{c}3,51 \mathrm{E}+ \\
04\end{array}$ & & $\begin{array}{c}5,71 \mathrm{E}+ \\
01\end{array}$ & $\begin{array}{c}3,86 \mathrm{E}+ \\
03\end{array}$ & $\begin{array}{c}4,10 \mathrm{E}+ \\
03\end{array}$ & $\begin{array}{c}1,75 \mathrm{E}+ \\
04\end{array}$ & & $\begin{array}{c}2,26 \mathrm{E}+ \\
02\end{array}$ & $\begin{array}{c}2,98 \mathrm{E}+ \\
03\end{array}$ & $\begin{array}{c}4,40 \mathrm{E}+ \\
03\end{array}$ & $\begin{array}{c}2,72 \mathrm{E}+ \\
04\end{array}$ & \\
\hline & $\begin{array}{c}\text { R- } \\
\text { EFO }\end{array}$ & $\begin{array}{c}1,40 \mathrm{E}+ \\
01\end{array}$ & $\begin{array}{c}5,21 \mathrm{E}+ \\
03\end{array}$ & $\begin{array}{c}5,53 \mathrm{E}+ \\
03\end{array}$ & $\begin{array}{c}2,02 \mathrm{E}+ \\
04\end{array}$ & $=$ & $\begin{array}{c}3,19 \mathrm{E}+ \\
01\end{array}$ & $\begin{array}{c}4,77 \mathrm{E}+ \\
03\end{array}$ & $\begin{array}{c}4,52 \mathrm{E}+ \\
03\end{array}$ & $\begin{array}{c}1,65 \mathrm{E}+ \\
04\end{array}$ & $=$ & $\begin{array}{c}7,53 \mathrm{E}+ \\
01\end{array}$ & $\begin{array}{c}2,05 \mathrm{E}+ \\
03\end{array}$ & $\begin{array}{c}2,34 \mathrm{E}+ \\
03\end{array}$ & $\begin{array}{c}1,02 \mathrm{E}+ \\
04\end{array}$ & $=$ \\
\hline \multirow{2}{*}{16} & EFO & $\begin{array}{c}2,31 \mathrm{E}+ \\
00\end{array}$ & $\begin{array}{c}3,38 \mathrm{E}+ \\
02\end{array}$ & $\begin{array}{c}3,38 \mathrm{E}+ \\
02\end{array}$ & $\begin{array}{c}1,29 \mathrm{E}+ \\
03\end{array}$ & & $\begin{array}{c}1,36 \mathrm{E}+ \\
02\end{array}$ & $\begin{array}{c}6,94 \mathrm{E}+ \\
02\end{array}$ & $\begin{array}{c}5,87 \mathrm{E}+ \\
02\end{array}$ & $\begin{array}{c}3,01 \mathrm{E}+ \\
03\end{array}$ & & $\begin{array}{c}4,96 \mathrm{E}+ \\
02\end{array}$ & $\begin{array}{c}2,19 \mathrm{E}+ \\
03\end{array}$ & $\begin{array}{c}1,54 \mathrm{E}+ \\
03\end{array}$ & $\begin{array}{c}7,66 \mathrm{E}+ \\
03\end{array}$ & \\
\hline & $\begin{array}{c}\text { R- } \\
\text { EFO }\end{array}$ & $\begin{array}{c}4,75 \mathrm{E}+ \\
00\end{array}$ & $\begin{array}{c}3,54 \mathrm{E}+ \\
02\end{array}$ & $\begin{array}{c}3,52 \mathrm{E}+ \\
02\end{array}$ & $\begin{array}{c}1,46 \mathrm{E}+ \\
03\end{array}$ & $=$ & $\begin{array}{c}2,43 \mathrm{E}+ \\
02\end{array}$ & $\begin{array}{c}6,23 \mathrm{E}+ \\
02\end{array}$ & $\begin{array}{c}3,81 \mathrm{E}+ \\
02\end{array}$ & $\begin{array}{c}2,51 \mathrm{E}+ \\
03\end{array}$ & $=$ & $\begin{array}{c}1,11 \mathrm{E}+ \\
03\end{array}$ & $\begin{array}{c}1,91 \mathrm{E}+ \\
03\end{array}$ & $\begin{array}{c}1,12 \mathrm{E}+ \\
03\end{array}$ & $\begin{array}{c}7,21 \mathrm{E}+ \\
03\end{array}$ & $=$ \\
\hline \multirow{2}{*}{17} & EFO & $\begin{array}{c}9,12 \mathrm{E}+ \\
00\end{array}$ & $\begin{array}{c}9,95 \mathrm{E}+ \\
01\end{array}$ & $\begin{array}{c}8,76 \mathrm{E}+ \\
01\end{array}$ & $\begin{array}{c}4,64 \mathrm{E}+ \\
02\end{array}$ & & $\begin{array}{c}7,88 \mathrm{E}+ \\
01\end{array}$ & $\begin{array}{c}8,10 \mathrm{E}+ \\
02\end{array}$ & $\begin{array}{c}6,15 \mathrm{E}+ \\
02\end{array}$ & $\begin{array}{c}2,18 \mathrm{E}+ \\
03\end{array}$ & & $\begin{array}{c}5,12 \mathrm{E}+ \\
02\end{array}$ & $\begin{array}{c}2,56 \mathrm{E}+ \\
03\end{array}$ & $\begin{array}{c}1,73 \mathrm{E}+ \\
03\end{array}$ & $\begin{array}{c}4,94 \mathrm{E}+ \\
03\end{array}$ & \\
\hline & $\begin{array}{c}\text { R- } \\
\text { EFO }\end{array}$ & $\begin{array}{c}2,97 \mathrm{E}+ \\
00\end{array}$ & $\begin{array}{c}7,66 \mathrm{E}+ \\
01\end{array}$ & $\begin{array}{c}8,31 \mathrm{E}+ \\
01\end{array}$ & $\begin{array}{c}3,25 \mathrm{E}+ \\
02\end{array}$ & + & $\begin{array}{c}5,15 \mathrm{E}+ \\
01\end{array}$ & $\begin{array}{c}7,84 \mathrm{E}+ \\
02\end{array}$ & $\begin{array}{c}5,81 \mathrm{E}+ \\
02\end{array}$ & $\begin{array}{c}1,87 \mathrm{E}+ \\
03\end{array}$ & $=$ & $\begin{array}{c}3,72 \mathrm{E}+ \\
02\end{array}$ & $\begin{array}{c}2,22 \mathrm{E}+ \\
03\end{array}$ & $\begin{array}{c}1,65 \mathrm{E}+ \\
03\end{array}$ & $\begin{array}{c}4,86 \mathrm{E}+ \\
03\end{array}$ & $=$ \\
\hline \multirow{2}{*}{18} & EFO & $\begin{array}{c}9,85 \mathrm{E}+ \\
03\end{array}$ & $\begin{array}{c}8,48 \mathrm{E}+ \\
04\end{array}$ & $\begin{array}{c}7,85 \mathrm{E}+ \\
04\end{array}$ & $\begin{array}{c}3,86 \mathrm{E}+ \\
05\end{array}$ & & $\begin{array}{c}7,06 \mathrm{E}+ \\
03\end{array}$ & $\begin{array}{c}3,41 \mathrm{E}+ \\
04\end{array}$ & $\begin{array}{c}2,16 \mathrm{E}+ \\
04\end{array}$ & $\begin{array}{c}9,85 \mathrm{E}+ \\
04\end{array}$ & & $\begin{array}{c}1,54 \mathrm{E}+ \\
05\end{array}$ & $\begin{array}{c}5,42 \mathrm{E}+ \\
05\end{array}$ & $\begin{array}{c}2,92 \mathrm{E}+ \\
05\end{array}$ & $\begin{array}{c}1,59 \mathrm{E}+ \\
06\end{array}$ & \\
\hline & $\begin{array}{c}\text { R- } \\
\text { EFO }\end{array}$ & $\begin{array}{c}1,09 \mathrm{E}+ \\
04\end{array}$ & $\begin{array}{c}1,20 \mathrm{E}+ \\
05\end{array}$ & $\begin{array}{c}1,08 \mathrm{E}+ \\
05\end{array}$ & $\begin{array}{c}5,15 \mathrm{E}+ \\
05\end{array}$ & $=$ & $\begin{array}{c}1,12 \mathrm{E}+ \\
04\end{array}$ & $\begin{array}{c}5,58 \mathrm{E}+ \\
04\end{array}$ & $\begin{array}{c}3,12 \mathrm{E}+ \\
04\end{array}$ & $\begin{array}{c}1,57 \mathrm{E}+ \\
05\end{array}$ & - & $\begin{array}{c}6,67 \mathrm{E}+ \\
04\end{array}$ & $\begin{array}{c}6,99 \mathrm{E}+ \\
05\end{array}$ & $\begin{array}{c}5,42 \mathrm{E}+ \\
05\end{array}$ & $\begin{array}{c}2,75 \mathrm{E}+ \\
06\end{array}$ & $=$ \\
\hline \multirow{2}{*}{19} & EFO & $\begin{array}{c}1,40 \mathrm{E}+ \\
01\end{array}$ & $\begin{array}{c}7,12 \mathrm{E}+ \\
03\end{array}$ & $\begin{array}{c}8,20 \mathrm{E}+ \\
03\end{array}$ & $\begin{array}{c}3,18 \mathrm{E}+ \\
04\end{array}$ & & $\begin{array}{c}4,64 \mathrm{E}+ \\
01\end{array}$ & $\begin{array}{c}1,28 \mathrm{E}+ \\
04\end{array}$ & $\begin{array}{c}9,20 \mathrm{E}+ \\
03\end{array}$ & $\begin{array}{c}3,95 \mathrm{E}+ \\
04\end{array}$ & & $\begin{array}{c}1,90 \mathrm{E}+ \\
02\end{array}$ & $\begin{array}{c}3,48 \mathrm{E}+ \\
03\end{array}$ & $\begin{array}{c}3,54 \mathrm{E}+ \\
03\end{array}$ & $\begin{array}{c}1,37 \mathrm{E}+ \\
04\end{array}$ & \\
\hline & $\begin{array}{c}\text { R- } \\
\text { EFO }\end{array}$ & $\begin{array}{c}1,30 \mathrm{E}+ \\
01\end{array}$ & $\begin{array}{c}9,48 \mathrm{E}+ \\
03\end{array}$ & $\begin{array}{c}1,17 \mathrm{E}+ \\
04\end{array}$ & $\begin{array}{c}4,76 \mathrm{E}+ \\
04\end{array}$ & $=$ & $\begin{array}{c}2,36 \mathrm{E}+ \\
01\end{array}$ & $\begin{array}{c}1,26 \mathrm{E}+ \\
04\end{array}$ & $\begin{array}{c}8,98 \mathrm{E}+ \\
03\end{array}$ & $\begin{array}{c}2,90 \mathrm{E}+ \\
04\end{array}$ & $=$ & $\begin{array}{c}7,70 \mathrm{E}+ \\
01\end{array}$ & $\begin{array}{c}2,38 \mathrm{E}+ \\
03\end{array}$ & $\begin{array}{c}3,44 \mathrm{E}+ \\
03\end{array}$ & $\begin{array}{c}1,34 \mathrm{E}+ \\
04\end{array}$ & + \\
\hline \multirow{2}{*}{20} & EFO & $\begin{array}{c}1,18 \mathrm{E}+ \\
01\end{array}$ & $\begin{array}{c}1,49 \mathrm{E}+ \\
02\end{array}$ & $\begin{array}{c}1,12 \mathrm{E}+ \\
02\end{array}$ & $\begin{array}{c}5,12 \mathrm{E}+ \\
02\end{array}$ & & $\begin{array}{c}4,49 \mathrm{E}+ \\
01\end{array}$ & $\begin{array}{c}5,18 \mathrm{E}+ \\
02\end{array}$ & $\begin{array}{c}5,88 \mathrm{E}+ \\
02\end{array}$ & $\begin{array}{c}1,70 \mathrm{E}+ \\
03\end{array}$ & & $\begin{array}{c}5,96 \mathrm{E}+ \\
02\end{array}$ & $\begin{array}{c}4,18 \mathrm{E}+ \\
03\end{array}$ & $\begin{array}{c}1,02 \mathrm{E}+ \\
03\end{array}$ & $\begin{array}{c}5,13 \mathrm{E}+ \\
03\end{array}$ & \\
\hline & $\begin{array}{c}\text { R- } \\
\text { EFO }\end{array}$ & $\begin{array}{c}5,17 \mathrm{E}+ \\
00\end{array}$ & $\begin{array}{c}1,15 \mathrm{E}+ \\
02\end{array}$ & $\begin{array}{c}7,98 \mathrm{E}+ \\
01\end{array}$ & $\begin{array}{c}3,51 \mathrm{E}+ \\
02\end{array}$ & + & $\begin{array}{c}3,61 \mathrm{E}+ \\
01\end{array}$ & $\begin{array}{c}4,55 \mathrm{E}+ \\
02\end{array}$ & $\begin{array}{c}5,25 \mathrm{E}+ \\
02\end{array}$ & $\begin{array}{c}1,65 \mathrm{E}+ \\
03\end{array}$ & $=$ & $\begin{array}{c}4,78 \mathrm{E}+ \\
02\end{array}$ & $\begin{array}{c}4,18 \mathrm{E}+ \\
03\end{array}$ & $\begin{array}{c}9,06 \mathrm{E}+ \\
02\end{array}$ & $\begin{array}{c}4,93 \mathrm{E}+ \\
03\end{array}$ & $=$ \\
\hline \multirow{2}{*}{21} & EFO & $\begin{array}{c}2,16 \mathrm{E}+ \\
02\end{array}$ & $\begin{array}{c}2,30 \mathrm{E}+ \\
02\end{array}$ & $\begin{array}{c}7,55 \mathrm{E}+ \\
00\end{array}$ & $\begin{array}{c}2,45 \mathrm{E}+ \\
02\end{array}$ & & $\begin{array}{c}2,49 \mathrm{E}+ \\
02\end{array}$ & $\begin{array}{c}2,71 \mathrm{E}+ \\
02\end{array}$ & $\begin{array}{c}1,45 \mathrm{E}+ \\
01\end{array}$ & $\begin{array}{c}3,25 \mathrm{E}+ \\
02\end{array}$ & & $\begin{array}{c}4,17 \mathrm{E}+ \\
02\end{array}$ & $\begin{array}{c}4,81 \mathrm{E}+ \\
02\end{array}$ & $\begin{array}{c}3,88 \mathrm{E}+ \\
01\end{array}$ & $\begin{array}{c}5,86 \mathrm{E}+ \\
02\end{array}$ & \\
\hline & $\begin{array}{c}\text { R- } \\
\text { EFO }\end{array}$ & $\begin{array}{c}2,14 \mathrm{E}+ \\
02\end{array}$ & $\begin{array}{c}2,29 \mathrm{E}+ \\
02\end{array}$ & $\begin{array}{c}2,25 \mathrm{E}+ \\
01\end{array}$ & $\begin{array}{c}3,50 \mathrm{E}+ \\
02\end{array}$ & + & $\begin{array}{c}2,40 \mathrm{E}+ \\
02\end{array}$ & $\begin{array}{c}2,63 \mathrm{E}+ \\
02\end{array}$ & $\begin{array}{c}1,12 \mathrm{E}+ \\
01\end{array}$ & $\begin{array}{c}2,91 \mathrm{E}+ \\
02\end{array}$ & + & $\begin{array}{c}3,93 \mathrm{E}+ \\
02\end{array}$ & $\begin{array}{c}4,55 \mathrm{E}+ \\
02\end{array}$ & $\begin{array}{c}3,23 \mathrm{E}+ \\
01\end{array}$ & $\begin{array}{c}5,50 \mathrm{E}+ \\
02\end{array}$ & + \\
\hline \multirow{2}{*}{22} & EFO & $\begin{array}{c}1,00 \mathrm{E}+ \\
02\end{array}$ & $\begin{array}{c}9,03 \mathrm{E}+ \\
02\end{array}$ & $\begin{array}{c}2,08 \mathrm{E}+ \\
03\end{array}$ & $\begin{array}{c}6,76 \mathrm{E}+ \\
03\end{array}$ & & $\begin{array}{c}1,00 \mathrm{E}+ \\
02\end{array}$ & $\begin{array}{c}1,17 \mathrm{E}+ \\
04\end{array}$ & $\begin{array}{c}3,04 \mathrm{E}+ \\
03\end{array}$ & $\begin{array}{c}1,36 \mathrm{E}+ \\
04\end{array}$ & & $\begin{array}{c}2,83 \mathrm{E}+ \\
04\end{array}$ & $\begin{array}{c}3,01 \mathrm{E}+ \\
04\end{array}$ & $\begin{array}{c}6,47 \mathrm{E}+ \\
02\end{array}$ & $\begin{array}{c}3,16 \mathrm{E}+ \\
04\end{array}$ & \\
\hline & $\begin{array}{c}\text { R- } \\
\text { EFO }\end{array}$ & $\begin{array}{c}1,00 \mathrm{E}+ \\
02\end{array}$ & $\begin{array}{c}6,94 \mathrm{E}+ \\
02\end{array}$ & $\begin{array}{c}1,83 \mathrm{E}+ \\
03\end{array}$ & $\begin{array}{c}\text { 7,03E+ } \\
03\end{array}$ & $=$ & $\begin{array}{c}1,00 \mathrm{E}+ \\
02\end{array}$ & $\begin{array}{c}1,25 \mathrm{E}+ \\
04\end{array}$ & $\begin{array}{c}1,85 \mathrm{E}+ \\
03\end{array}$ & $\begin{array}{c}1,37 \mathrm{E}+ \\
04\end{array}$ & $=$ & $\begin{array}{c}2,79 \mathrm{E}+ \\
04\end{array}$ & $\begin{array}{c}3,00 \mathrm{E}+ \\
04\end{array}$ & $\begin{array}{c}\text { 7,57E+ } \\
02\end{array}$ & $\begin{array}{c}3,13 \mathrm{E}+ \\
04\end{array}$ & $=$ \\
\hline \multirow{2}{*}{23} & EFO & $\begin{array}{c}3,64 \mathrm{E}+ \\
02\end{array}$ & $\begin{array}{c}3,88 \mathrm{E}+ \\
02\end{array}$ & $\begin{array}{c}1,32 \mathrm{E}+ \\
01\end{array}$ & $\begin{array}{c}4,35 \mathrm{E}+ \\
02\end{array}$ & & $\begin{array}{c}4,79 \mathrm{E}+ \\
02\end{array}$ & $\begin{array}{c}5,28 \mathrm{E}+ \\
02\end{array}$ & $\begin{array}{c}2,68 \mathrm{E}+ \\
01\end{array}$ & $\begin{array}{c}5,83 \mathrm{E}+ \\
02\end{array}$ & & $\begin{array}{c}8,21 \mathrm{E}+ \\
02\end{array}$ & $\begin{array}{c}9,48 \mathrm{E}+ \\
02\end{array}$ & $\begin{array}{c}6,93 \mathrm{E}+ \\
01\end{array}$ & $\begin{array}{c}1,18 \mathrm{E}+ \\
03\end{array}$ & \\
\hline & $\begin{array}{c}\text { R- } \\
\text { EFO }\end{array}$ & $\begin{array}{c}3,59 \mathrm{E}+ \\
02\end{array}$ & $\begin{array}{c}3,82 \mathrm{E}+ \\
02\end{array}$ & $\begin{array}{c}1,14 \mathrm{E}+ \\
01\end{array}$ & $\begin{array}{c}4,28 \mathrm{E}+ \\
02\end{array}$ & + & $\begin{array}{c}4,61 \mathrm{E}+ \\
02\end{array}$ & $\begin{array}{c}5,09 \mathrm{E}+ \\
02\end{array}$ & $\begin{array}{c}2,02 \mathrm{E}+ \\
01\end{array}$ & $\begin{array}{c}5,54 \mathrm{E}+ \\
02\end{array}$ & + & $\begin{array}{c}6,86 \mathrm{E}+ \\
02\end{array}$ & $\begin{array}{c}\text { 7,72E+ } \\
02\end{array}$ & $\begin{array}{c}4,56 \mathrm{E}+ \\
01\end{array}$ & $\begin{array}{c}8,85 \mathrm{E}+ \\
02\end{array}$ & + \\
\hline \multirow{2}{*}{24} & EFO & $\begin{array}{c}4,26 \mathrm{E}+ \\
02\end{array}$ & $\begin{array}{c}4,63 \mathrm{E}+ \\
02\end{array}$ & $\begin{array}{c}3,84 \mathrm{E}+ \\
01\end{array}$ & $\begin{array}{c}5,98 \mathrm{E}+ \\
02\end{array}$ & & $\begin{array}{c}5,46 \mathrm{E}+ \\
02\end{array}$ & $\begin{array}{c}5,91 \mathrm{E}+ \\
02\end{array}$ & $\begin{array}{c}4,22 \mathrm{E}+ \\
01\end{array}$ & $\begin{array}{c}8,36 \mathrm{E}+ \\
02\end{array}$ & & $\begin{array}{c}1,25 \mathrm{E}+ \\
03\end{array}$ & $\begin{array}{c}1,44 \mathrm{E}+ \\
03\end{array}$ & $\begin{array}{c}1,08 \mathrm{E}+ \\
02\end{array}$ & $\begin{array}{c}1,67 \mathrm{E}+ \\
03\end{array}$ & \\
\hline & $\begin{array}{c}\text { R- } \\
\text { EFO }\end{array}$ & $\begin{array}{c}4,38 \mathrm{E}+ \\
02\end{array}$ & $\begin{array}{c}4,70 \mathrm{E}+ \\
02\end{array}$ & $\begin{array}{c}5,15 \mathrm{E}+ \\
01\end{array}$ & $\begin{array}{c}6,00 \mathrm{E}+ \\
02\end{array}$ & $=$ & $\begin{array}{c}5,32 \mathrm{E}+ \\
02\end{array}$ & $\begin{array}{c}5,83 \mathrm{E}+ \\
02\end{array}$ & $\begin{array}{c}6,88 \mathrm{E}+ \\
01\end{array}$ & $\begin{array}{c}8,74 \mathrm{E}+ \\
02\end{array}$ & + & $\begin{array}{c}1,16 \mathrm{E}+ \\
03\end{array}$ & $\begin{array}{c}1,29 \mathrm{E}+ \\
03\end{array}$ & $\begin{array}{c}6,72 \mathrm{E}+ \\
01\end{array}$ & $\begin{array}{c}1,44 \mathrm{E}+ \\
03\end{array}$ & + \\
\hline \multirow{2}{*}{25} & EFO & $\begin{array}{c}3,84 \mathrm{E}+ \\
02\end{array}$ & $\begin{array}{c}3,92 \mathrm{E}+ \\
02\end{array}$ & $\begin{array}{c}1,22 \mathrm{E}+ \\
01\end{array}$ & $\begin{array}{c}4,41 \mathrm{E}+ \\
02\end{array}$ & & $\begin{array}{c}4,61 \mathrm{E}+ \\
02\end{array}$ & $\begin{array}{c}5,46 \mathrm{E}+ \\
02\end{array}$ & $\begin{array}{c}3,60 \mathrm{E}+ \\
01\end{array}$ & $\begin{array}{c}5,98 \mathrm{E}+ \\
02\end{array}$ & & $\begin{array}{c}6,19 \mathrm{E}+ \\
02\end{array}$ & $\begin{array}{c}7,78 \mathrm{E}+ \\
02\end{array}$ & $\begin{array}{c}7,12 \mathrm{E}+ \\
01\end{array}$ & $\begin{array}{c}9,20 \mathrm{E}+ \\
02\end{array}$ & \\
\hline & $\begin{array}{c}\text { R- } \\
\text { EFO }\end{array}$ & $\begin{array}{c}3,83 \mathrm{E}+ \\
02\end{array}$ & $\begin{array}{c}3,92 \mathrm{E}+ \\
02\end{array}$ & $\begin{array}{c}1,33 \mathrm{E}+ \\
01\end{array}$ & $\begin{array}{c}4,42 \mathrm{E}+ \\
02\end{array}$ & $=$ & $\begin{array}{c}4,28 \mathrm{E}+ \\
02\end{array}$ & $\begin{array}{c}5,27 \mathrm{E}+ \\
02\end{array}$ & $\begin{array}{c}4,62 \mathrm{E}+ \\
01\end{array}$ & $\begin{array}{c}6,03 \mathrm{E}+ \\
02\end{array}$ & + & $\begin{array}{c}6,21 \mathrm{E}+ \\
02\end{array}$ & $\begin{array}{c}7,72 \mathrm{E}+ \\
02\end{array}$ & $\begin{array}{c}7,48 \mathrm{E}+ \\
01\end{array}$ & $\begin{array}{c}9,42 \mathrm{E}+ \\
02\end{array}$ & $=$ \\
\hline \multirow{2}{*}{26} & EFO & $\begin{array}{c}2,00 \mathrm{E}+ \\
02\end{array}$ & $\begin{array}{c}1,51 \mathrm{E}+ \\
03\end{array}$ & $\begin{array}{c}2,74 \mathrm{E}+ \\
02\end{array}$ & $\begin{array}{c}1,95 \mathrm{E}+ \\
03\end{array}$ & & $\begin{array}{c}1,66 \mathrm{E}+ \\
03\end{array}$ & $\begin{array}{c}2,47 \mathrm{E}+ \\
03\end{array}$ & $\begin{array}{c}3,37 \mathrm{E}+ \\
02\end{array}$ & $\begin{array}{c}3,20 \mathrm{E}+ \\
03\end{array}$ & & $\begin{array}{c}6,96 \mathrm{E}+ \\
03\end{array}$ & $\begin{array}{c}8,79 \mathrm{E}+ \\
03\end{array}$ & $\begin{array}{c}9,36 \mathrm{E}+ \\
02\end{array}$ & $\begin{array}{c}1,12 \mathrm{E}+ \\
04\end{array}$ & \\
\hline & $\begin{array}{c}\text { R- } \\
\text { EFO }\end{array}$ & $\begin{array}{c}1,13 \mathrm{E}+ \\
03\end{array}$ & $\begin{array}{c}1,39 \mathrm{E}+ \\
03\end{array}$ & $\begin{array}{c}1,62 \mathrm{E}+ \\
02\end{array}$ & $\begin{array}{c}1,82 \mathrm{E}+ \\
03\end{array}$ & + & $\begin{array}{c}1,81 \mathrm{E}+ \\
03\end{array}$ & $\begin{array}{c}2,25 \mathrm{E}+ \\
03\end{array}$ & $\begin{array}{c}2,56 \mathrm{E}+ \\
02\end{array}$ & $\begin{array}{c}2,82 \mathrm{E}+ \\
03\end{array}$ & + & $\begin{array}{c}5,96 \mathrm{E}+ \\
03\end{array}$ & $\begin{array}{c}7,29 \mathrm{E}+ \\
03\end{array}$ & $\begin{array}{c}7,66 \mathrm{E}+ \\
02\end{array}$ & $\begin{array}{c}9,04 \mathrm{E}+ \\
03\end{array}$ & + \\
\hline \multirow{2}{*}{27} & EFO & $\begin{array}{c}5,08 \mathrm{E}+ \\
02\end{array}$ & $\begin{array}{c}5,34 \mathrm{E}+ \\
02\end{array}$ & $\begin{array}{c}1,56 \mathrm{E}+ \\
01\end{array}$ & $\begin{array}{c}5,76 \mathrm{E}+ \\
02\end{array}$ & & $\begin{array}{c}6,20 \mathrm{E}+ \\
02\end{array}$ & $\begin{array}{c}7,80 \mathrm{E}+ \\
02\end{array}$ & $\begin{array}{c}7,05 \mathrm{E}+ \\
01\end{array}$ & $\begin{array}{c}9,69 \mathrm{E}+ \\
02\end{array}$ & & $\begin{array}{c}8,33 \mathrm{E}+ \\
02\end{array}$ & $\begin{array}{c}1,01 \mathrm{E}+ \\
03\end{array}$ & $\begin{array}{c}1,04 \mathrm{E}+ \\
02\end{array}$ & $\begin{array}{c}1,34 \mathrm{E}+ \\
03\end{array}$ & \\
\hline & $\begin{array}{c}\text { R- } \\
\text { EFO }\end{array}$ & $\begin{array}{c}5,07 \mathrm{E}+ \\
02\end{array}$ & $\begin{array}{c}5,29 \mathrm{E}+ \\
02\end{array}$ & $\begin{array}{c}1,33 \mathrm{E}+ \\
01\end{array}$ & $\begin{array}{c}5,61 \mathrm{E}+ \\
02\end{array}$ & $=$ & $\begin{array}{c}5,87 \mathrm{E}+ \\
02\end{array}$ & $\begin{array}{c}7,06 \mathrm{E}+ \\
02\end{array}$ & $\begin{array}{c}6,36 \mathrm{E}+ \\
01\end{array}$ & $\begin{array}{c}8,68 \mathrm{E}+ \\
02\end{array}$ & + & $\begin{array}{c}7,83 \mathrm{E}+ \\
02\end{array}$ & $\begin{array}{c}8,97 \mathrm{E}+ \\
02\end{array}$ & $\begin{array}{c}6,85 \mathrm{E}+ \\
01\end{array}$ & $\begin{array}{c}1,09 \mathrm{E}+ \\
03\end{array}$ & + \\
\hline \multirow{2}{*}{28} & EFO & $\begin{array}{c}3,00 \mathrm{E}+ \\
02\end{array}$ & $\begin{array}{c}3,40 \mathrm{E}+ \\
02\end{array}$ & $\begin{array}{c}5,30 \mathrm{E}+ \\
01\end{array}$ & $\begin{array}{c}4,14 \mathrm{E}+ \\
02\end{array}$ & & $\begin{array}{c}4,53 \mathrm{E}+ \\
02\end{array}$ & $\begin{array}{c}4,92 \mathrm{E}+ \\
02\end{array}$ & $\begin{array}{c}2,27 \mathrm{E}+ \\
01\end{array}$ & $\begin{array}{c}5,89 \mathrm{E}+ \\
02\end{array}$ & & $\begin{array}{c}3,00 \mathrm{E}+ \\
02\end{array}$ & $\begin{array}{c}5,03 \mathrm{E}+ \\
02\end{array}$ & $\begin{array}{c}5,63 \mathrm{E}+ \\
01\end{array}$ & $\begin{array}{c}6,29 \mathrm{E}+ \\
02\end{array}$ & \\
\hline & $\begin{array}{c}\text { R- } \\
\text { EFO }\end{array}$ & $\begin{array}{c}3,00 \mathrm{E}+ \\
02\end{array}$ & $\begin{array}{c}3,30 \mathrm{E}+ \\
02\end{array}$ & $\begin{array}{c}5,19 \mathrm{E}+ \\
01\end{array}$ & $\begin{array}{c}4,60 \mathrm{E}+ \\
02\end{array}$ & $=$ & $\begin{array}{c}4,59 \mathrm{E}+ \\
02\end{array}$ & $\begin{array}{c}4,96 \mathrm{E}+ \\
02\end{array}$ & $\begin{array}{c}2,63 \mathrm{E}+ \\
01\end{array}$ & $\begin{array}{c}5,88 \mathrm{E}+ \\
02\end{array}$ & $=$ & $\begin{array}{c}4,27 \mathrm{E}+ \\
02\end{array}$ & $\begin{array}{c}5,38 \mathrm{E}+ \\
02\end{array}$ & $\begin{array}{c}3,86 \mathrm{E}+ \\
01\end{array}$ & $\begin{array}{c}6,36 \mathrm{E}+ \\
02\end{array}$ & \\
\hline 29 & $\mathbf{E}$ & $\begin{array}{c}4,40 \mathrm{E}+ \\
02\end{array}$ & $\begin{array}{c}6,24 \mathrm{E}+ \\
02\end{array}$ & $\begin{array}{c}1,20 \mathrm{E}+ \\
02\end{array}$ & $\begin{array}{c}8,63 \mathrm{E}+ \\
02\end{array}$ & & $\begin{array}{c}3,85 \mathrm{E}+ \\
02\end{array}$ & $\begin{array}{c}7,99 \mathrm{E}+ \\
02\end{array}$ & $\begin{array}{c}2,30 \mathrm{E}+ \\
02\end{array}$ & $\begin{array}{c}1,32 \mathrm{E}+ \\
03\end{array}$ & & $\begin{array}{c}1,89 \mathrm{E}+ \\
03\end{array}$ & $\begin{array}{c}2,71 \mathrm{E}+ \\
03\end{array}$ & $\begin{array}{c}4,11 \mathrm{E}+ \\
02\end{array}$ & $\begin{array}{c}3,79 \mathrm{E}+ \\
03\end{array}$ & \\
\hline
\end{tabular}


Tablo 1 (devam). EFO ve R-EFO algoritmalarının Wilcoxon karşılaştırma sonuçları

\begin{tabular}{|c|c|c|c|c|c|c|c|c|c|c|c|c|c|c|c|c|}
\hline & $\begin{array}{c}\text { R- } \\
\text { EFO }\end{array}$ & $\begin{array}{c}3,67 \mathrm{E}+ \\
02\end{array}$ & $\begin{array}{c}5,46 \mathrm{E}+ \\
02\end{array}$ & $\begin{array}{c}1,09 \mathrm{E}+ \\
02\end{array}$ & $\begin{array}{c}8,49 \mathrm{E}+ \\
02\end{array}$ & + & $\begin{array}{c}3,35 \mathrm{E}+ \\
02\end{array}$ & $\begin{array}{c}6,30 \mathrm{E}+ \\
02\end{array}$ & $\begin{array}{c}1,73 \mathrm{E}+ \\
02\end{array}$ & $\begin{array}{c}1,13 \mathrm{E}+ \\
03\end{array}$ & + & $\begin{array}{c}1,57 \mathrm{E}+ \\
03\end{array}$ & $\begin{array}{c}2,28 \mathrm{E}+ \\
03\end{array}$ & $\begin{array}{c}4,44 \mathrm{E}+ \\
02\end{array}$ & $\begin{array}{c}3,47 \mathrm{E}+ \\
03\end{array}$ & ${ }^{+}$ \\
\hline \multirow[b]{2}{*}{30} & EFO & $\begin{array}{c}2,33 \mathrm{E}+ \\
03\end{array}$ & $\begin{array}{c}4,67 \mathrm{E}+ \\
03\end{array}$ & $\begin{array}{c}1,96 \mathrm{E}+ \\
03\end{array}$ & $\begin{array}{c}1,16 \mathrm{E}+ \\
04\end{array}$ & & $\begin{array}{c}6,49 \mathrm{E}+ \\
05\end{array}$ & $\begin{array}{c}8,04 \mathrm{E}+ \\
05\end{array}$ & $\begin{array}{c}8,96 \mathrm{E}+ \\
04\end{array}$ & $\begin{array}{c}1,03 \mathrm{E}+ \\
06\end{array}$ & & $\begin{array}{c}2,84 \mathrm{E}+ \\
03\end{array}$ & $\begin{array}{c}6,38 \mathrm{E}+ \\
03\end{array}$ & $\begin{array}{c}2,63 \mathrm{E}+ \\
03\end{array}$ & $\begin{array}{c}1,46 \mathrm{E}+ \\
04\end{array}$ & \\
\hline & $\begin{array}{c}\text { R- } \\
\text { EFO }\end{array}$ & $\begin{array}{c}2,30 \mathrm{E}+ \\
03\end{array}$ & $\begin{array}{c}5,08 \mathrm{E}+ \\
03\end{array}$ & $\begin{array}{c}2,36 \mathrm{E}+ \\
03\end{array}$ & $\begin{array}{c}1,14 \mathrm{E}+ \\
04\end{array}$ & $=$ & $\begin{array}{c}6,21 \mathrm{E}+ \\
05\end{array}$ & $\begin{array}{c}8,14 \mathrm{E}+ \\
05\end{array}$ & $\begin{array}{c}9,71 \mathrm{E}+ \\
04\end{array}$ & $\begin{array}{c}1,05 \mathrm{E}+ \\
06\end{array}$ & $=$ & $\begin{array}{c}2,78 \mathrm{E}+ \\
03\end{array}$ & $\begin{array}{c}6,27 \mathrm{E}+ \\
03\end{array}$ & $\begin{array}{c}3,11 \mathrm{E}+ \\
03\end{array}$ & $\begin{array}{c}1,47 \mathrm{E}+ \\
04\end{array}$ & $=$ \\
\hline \multicolumn{2}{|c|}{ Wilcoxon } & \multicolumn{6}{|c|}{$13 / 16 / 1$} & \multicolumn{6}{|c|}{$15 / 11 / 4$} & \multicolumn{2}{|c|}{$15 / 10 / 5$} & \\
\hline
\end{tabular}

\section{IV.SONUC}

$\mathrm{Bu}$ makale çalışmasında rulet seçim yönteminin EFO algoritmasının arama performansını artırdığı görülmüştür. R-EFO algoritmasında üç alt popülasyondan rastgele seçilen çözüm adaylarının ikisi EFO algoritmasındakiyle aynı kalırken negatif topluluktan seçim sürecinde rulet olasılıksal seçim yöntemini kullanmanın arama sürecinde daha etkili bir rehberlik yaptığı görülmüştür. $\mathrm{Bu}$ etki R-EFO algoritmasının komşuluk araması ve çeşitlilik yeteneklerinin EFO algoritmasına kıyasla daha güçlü olduğuna işaret etmektedir. Üstelik problem boyutu arttıkça R-EFO algoritmasının performansında kararl1l1k olduğu görülmektedir.

\section{KAYNAKLAR}

[1] Abedinpourshotorban, H., Shamsuddin, S. M., Beheshti, Z., \& Jawawi, D. N. "Electromagnetic field optimization: A physics-inspired metaheuristic optimization algorithm", Swarm and Evolutionary Computation, no. 26, pp. 8-22, 2016.

[2] Al-Bahrani, L. T., \& Patra, J. C. "A novel orthogonal PSO algorithm based on orthogonal diagonalization", Swarm and Evolutionary Computation, no. 40, pp. 1-23,2018

[3] Ali, A. F., Tawhid, M. A. "A hybrid particle swarm optimization and genetic algorithm with population partitioning for large scale optimization problems", Ain Shams Engineering Journal, vol.8, no. 2, pp. 191-206, 2017.

[4] Awad, N. H., Ali, M. Z., Mallipeddi, R., \& Suganthan, P. N. "An improved differential evolution algorithm using efficient adapted surrogate model for numerical optimization," Information Sciences, 451, 326-347, 2018.

[5] Aydilek, İ. B. "A hybrid firefly and particle swarm optimization algorithm for computationally expensive numerical problems", Applied Soft Computing, 66, 232-249, 2018.

[6] Caraveo, C., Valdez, F., \& Castillo, O. "A new optimization meta-heuristic algorithm based on self-defense mechanism of the plants with three reproduction operators", Soft Computing, pp.1-14, 2018.

[7] Chen, K., Zhou, F., Yin, L., Wang, S., Wang, Y., Wan, F. "A hybrid particle swarm optimizer with sine cosine acceleration coefficients", Information Sciences, 422, pp. 218-241, 2018.

[8] Cheng, M. Y., \& Prayogo, D. "Symbiotic organisms search: a new metaheuristic optimization algorithm," Computers \& Structures, 139, pp. 98-112, 2014. 
[9] Cheraghalipour, A., Hajiaghaei-Keshteli, M., \& Paydar, M. M. 2018. "Tree Growth Algorithm (TGA): A novel approach for solving optimization problems", Engineering Applications of Artificial Intelligence, 72, 393-414.

[10] Holland, J.H., 1975. "Adaptation in natural and artificial systems: An introductory analysis with applications to biology, control, and artificial intelligence". Q. Rev. Biol. 1, 211. http://dx.doi.org/10.1086/418447.

[11] Hooker, J. N. "Testing heuristics: We have it all wrong," Journal of heuristics, vol. 1, no. 1, pp. $33-42,1995$.

[12] Kahraman, H. T., Aras, S., Guvenc, U., \& Sonmez, Y. "Exploring the effect of distribution methods on meta-heuristic searching process". International Conference on Computer Science and Engineering (UBMK), Antalya, 5-8 Ekim 2017, IEEE, pp. 371-376, 2017.

[13] Kahraman, H. T., Aras, S., Gedikli, E. "Meta-Sezgisel Optimizasyon Çalışmalarında Benchmark Problemlerinde Karşılaşılan Standartsızlıklar ve Çözüm Önerileri", IV. INES Internatıonal Academic Research Congress (INES-2018), Antalya, 30 Ekim-3 Kasim 2018.

[14] Kahraman, H. T., Aras, S., Gedikli, E., "Meta-Sezgisel Algoritmaların Deneysel Çalışmalarındaki Standartsızlıklar ve Çözüm Önerileri", , IV. INES Internatıonal Academic Research Congress (INES - 2018), Antalya, 30 Ekim-3 Kasım 2018.

[15] Cui, L., Li, G., Zhu, Z., Lin, Q., Wong, K. C., Chen, J., Lu, J. "Adaptive multiple-elites-guided composite differential evolution algorithm with a shift mechanism", Information Sciences, 422, 122 $143,2018$.

[16] Awad, N. H., Ali, M. Z., Liang, J. J., Qu, B. Y., \& Suganthan, P. N. (2016). "Problem definitions and evaluation criteria for the CEC 2017 special session and competition on single objective realparameter numerical optimization. Tech. Rep.", (son erişim tarihi: 1.07.2019).

[17] Liang, J.J., Qu, B.Y., Suganthan, P.N. "Problem Definitions and Evaluation Criteria for the CEC 2014 Special Session and Competition on Single Objective Real-Parameter Numerical Optimization", Computational Intelligence Laboratory, Zhengzhou University, Zhengzhou, China and Nanyang Technological University, Singapore, 2013.

[18] Le, D. T., Bui, D. K., Ngo, T. D., Nguyen, Q. H., \& Nguyen-Xuan, H. (2019). “A novel hybrid method combining electromagnetism-like mechanism and firefly algorithms for constrained design optimization of discrete truss structures", Computers \& Structures, 212, 20-42.

[19] Mirjalili, S., Gandomi, A. H., Mirjalili, S. Z., Saremi, S., Faris, H., \& Mirjalili, S. M. "Salp Swarm Algorithm: A bio-inspired optimizer for engineering design problems", Advances in Engineering Software, 114, pp. 163-191, 2017.

[20] Wang, L., Yang, B., \& Orchard, J. "Particle swarm optimization using dynamic tournament topology," Applied Soft Computing, 48, 584-596, 2016.

[21] Qin, Q., Cheng, S., Zhang, Q., Li, L., \& Shi, Y. "Particle swarm optimization with interswarm interactive learning strategy", IEEE transactions on cybernetics, vol. 46, no.10, pp. 2238-2251, 2016.

[22] Al-Bahrani, L. T., \& Patra, J. C. "A novel orthogonal PSO algorithm based on orthogonal diagonalization," Swarm and Evolutionary Computation, 40, pp. 1-23, 2018. 
[23] Lin, Q., Zhu, M., Li, G., Wang, W., Cui, L., Chen, J., \& Lu, J. "A novel artificial bee colony algorithm with local and global information interaction", Applied Soft Computing, 62, 702-735, 2018.

[24] Long, W., Jiao, J., Liang, X., Tang, M. "An exploration-enhanced grey wolf optimizer to solve high-dimensional numerical optimization", Engineering Applications of Artificial Intelligence, 68, pp. 63-80, 2018.

[25] Mirjalili, S., Gandomi, A. H., Mirjalili, S. Z., Saremi, S., Faris, H., \& Mirjalili, S. M. "Salp Swarm Algorithm: A bio-inspired optimizer for engineering design problems". Advances in Engineering Software, 114, pp. 163-191, 2017.

[26] Salgotra, R., Singh, U., \& Saha, S. "New cuckoo search algorithms with enhanced exploration and exploitation properties", Expert Systems with Applications, 95, 384-420, 2018.

[27] Sun, G., Ma, P., Ren, J., Zhang, A., \& Jia, X. "A stability constrained adaptive alpha for gravitational search algorithm," Knowledge-Based Systems, 139, pp. 200-213, 2018.

[28] Torabi, S., \& Safi-Esfahani, F. "Improved Raven Roosting Optimization algorithm (IRRO)", Swarm and Evolutionary Computation, 40, pp. 144-154, 2018.

[29] Veçek, N., Mernik, M., \& Črepinšek, M. "A chess rating system for evolutionary algorithms: A new method for the comparison and ranking of evolutionary algorithms," Information Sciences, 277, pp. 656-679, 2014.

[30] Wu, G., Shen, X., Li, H., Chen, H., Lin, A., Suganthan, P. N. "Ensemble of differential evolution variants", Information Sciences, 423, pp. 172-186, 2018. 